\title{
Association of Diet Quality and Body Mass Index with Serum Ceramides in Middle-aged Adults
}

\author{
Margaret Ann Drazba \\ West Virginia University, madrazba@mix.wvu.edu
}

Follow this and additional works at: https://researchrepository.wvu.edu/etd

Part of the Dietetics and Clinical Nutrition Commons

\section{Recommended Citation}

Drazba, Margaret Ann, "Association of Diet Quality and Body Mass Index with Serum Ceramides in Middleaged Adults" (2018). Graduate Theses, Dissertations, and Problem Reports. 3708.

https://researchrepository.wvu.edu/etd/3708

This Thesis is protected by copyright and/or related rights. It has been brought to you by the The Research Repository @ WVU with permission from the rights-holder(s). You are free to use this Thesis in any way that is permitted by the copyright and related rights legislation that applies to your use. For other uses you must obtain permission from the rights-holder(s) directly, unless additional rights are indicated by a Creative Commons license in the record and/ or on the work itself. This Thesis has been accepted for inclusion in WVU Graduate Theses, Dissertations, and Problem Reports collection by an authorized administrator of The Research Repository @ WVU. For more information, please contact researchrepository@mail.wvu.edu. 


\title{
Association of Diet Quality and Body Mass Index with Serum Ceramides in Middle-aged Adults
}

\author{
Margaret Ann Drazba
}

Thesis submitted to the Davis College of Agriculture, Natural Resources, and Design at West Virginia University

in partial fulfillment of the requirements for the degree of

\author{
Master of Science \\ in Nutrition and Food Science
}

Melissa Ventura-Marra, $\mathrm{PhD}, \mathrm{RD}$, Committee Chair

Nadine Sahyoun, $\mathrm{PhD}, \mathrm{RD}$

Ida Holásková, $\mathrm{PhD}$

Department of Animal and Nutritional Sciences

Morgantown, West Virginia

2018

Key words: diet quality, healthy eating index, ceramides, obesity, cardiovascular risk Copyright 2018 Margaret Drazba 


\section{ABSTRACT \\ Association of Diet Quality and Body Mass Index with Serum Ceramides in Middle-Aged Adults}

\section{Margaret Ann Drazba}

Cardiovascular disease (CVD) is one of the leading causes of death worldwide and identifying CVD risk is important for determining and implementing appropriate intervention strategies. Ceramides are a class of sphingolipids that are implicated in the pathogenesis of obesity and CVD. However, their relationship to diet quality, a modifiable risk factor for CVD, is largely unknown. The purpose of this cross-sectional study was to determine the association of an $a$ priori diet quality index and body mass index (BMI) with circulating ceramides in middle-aged adults ( $\mathrm{n}=96)$. Dietary intake was assessed using three 24-hour recalls and Nutrition Data System for Research (NDSR) software. Diet quality was estimated using the Healthy Eating Index (HEI2015). Serum ceramide concentrations were determined by quantitative liquid chromatography mass spectrometry (LC/MS). A ceramide risk score was calculated based on published metrics. After adjusting for confounding cardiovascular risk factors, BMI $\left(\mathrm{kg} / \mathrm{m}^{2}\right)$ was positively associated with $\mathrm{C} 18: 0\left(\mathrm{R}^{2}=0.31, \mathrm{p}<0.0001\right), \mathrm{C} 18: 0 / \mathrm{C} 24: 0\left(\mathrm{R}^{2}=0.30, \mathrm{p}<0.0001\right)$ and the ceramide risk score $\left(\mathrm{R}^{2}=0.11, \mathrm{p}<0.009\right)$. HEI-2015 was inversely associated with $\mathrm{C} 22: 0\left(\mathrm{R}^{2}=0.42\right.$, $\mathrm{p}=0.02$ ). These findings suggest that BMI is more predictive of ceramide concentration than diet quality as measured by the HEI-2015 in this sample. Studies to determine if reduction in weight status results in lower ceramide risk scores in humans are needed. 


\section{ACKNOWLEDGEMENTS}

I would like to acknowledge and thank several people who were instrumental in the completion of this thesis. First, I want to recognize and thank Dr. Melissa Marra for her endless support and guidance as my advisor and mentor over the past four years. You have positively impacted my academic career since I was an undergraduate student, and I will truly miss working with you. I would also like to thank my committee members, Dr. Ida Holásková and Dr. Nadine Sahyoun, for their invaluable feedback and assistance with my project. Additionally, many faculty members at WVU have provided me with support and guidance throughout my undergraduate and graduate careers, and I would like to extend my sincere thanks to you. I would also like to thank the past researchers of Dr. Marra's lab for your contributions to data collection in the West Virginia Diet and Health Assessment Study, without which my thesis would not be possible. Finally, I want to thank my family and friends for their support of my academic endeavors. I would not be where I am today without each of you.

This project was generously funded by the WVU Provost's Office, Health Disparities Mountains of Excellence flash fund award; the USDA National Institute of Food and Agriculture (NIFA) Hatch/Multi-State project 1009924 (WVU) and the West Virginia Agricultural and Forestry Experiment Station. Software was provided by the West Virginia Clinical and Translational Science Institute (National Institute of General Medical Sciences of the National Institutes of Health under Award Number 2U54GM104942-02). 


\section{PREFACE}

This thesis was written in the format as prescribed by West Virginia University Electronic Thesis Document guidelines. This thesis document contains one manuscript:

Association of Diet Quality and Body Mass Index with Serum Ceramides in Middle-aged Adults with at Least One Risk Factor for Cardiovascular Disease. The manuscript has been formatted for publication in Nutrients. 


\section{TABLE OF CONTENTS}

ABSTRACT

Error! Bookmark not defined.

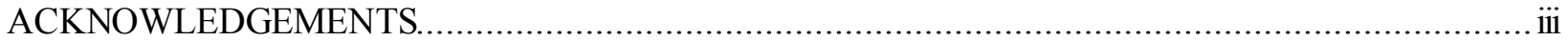

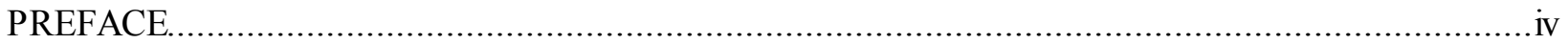

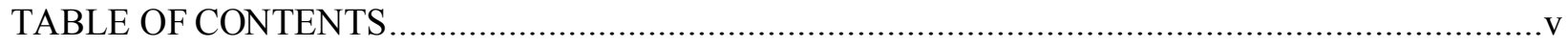

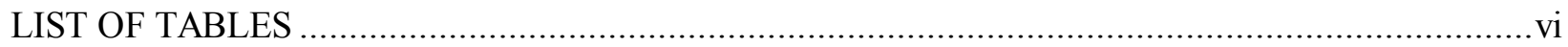

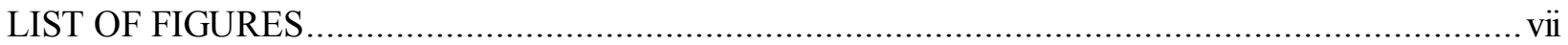

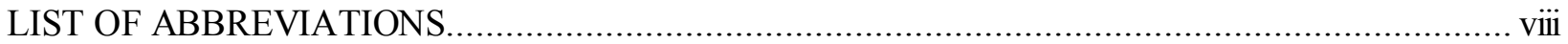

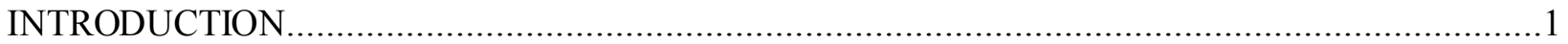

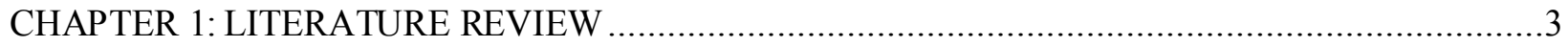

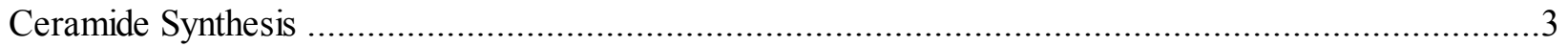

Serum Ceramides as a Risk Factor for Cardiovascular Disease ................................................5

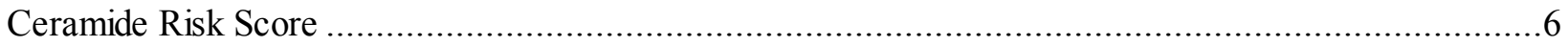

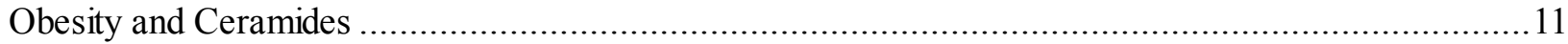

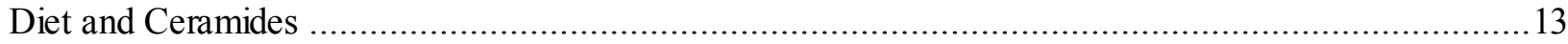

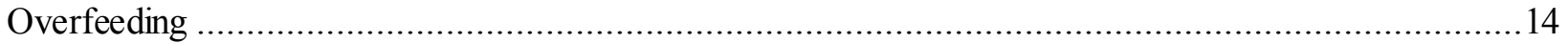

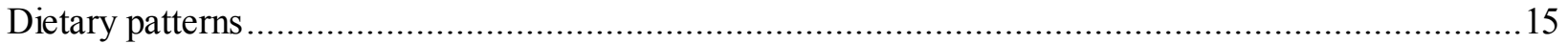

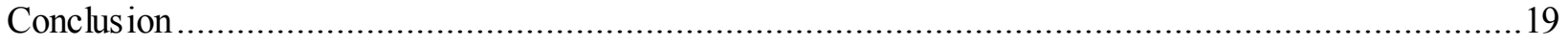

REFERENCES.

CHAPTER 2: Association of Diet Quality and Body Mass Index with Serum Ceramides in Middle-aged Adults with at Least One Risk Factor for Cardiovascular Disease ..............................................24

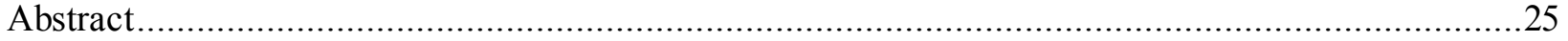

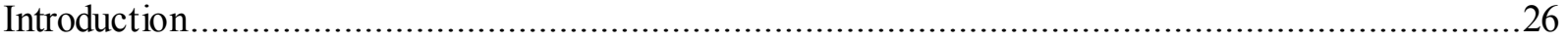

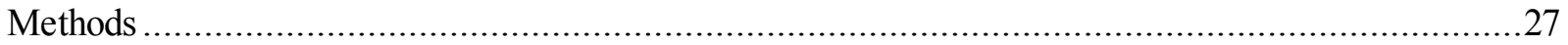

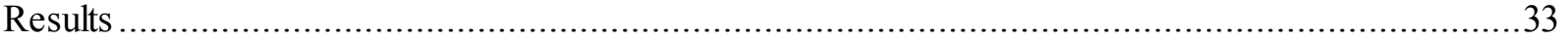

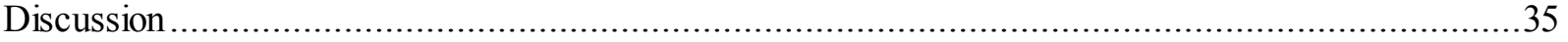

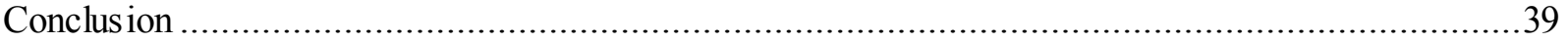

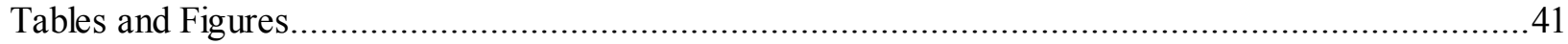

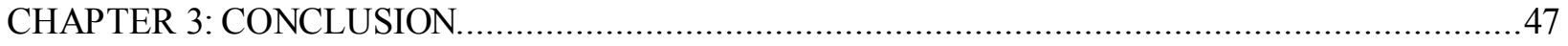

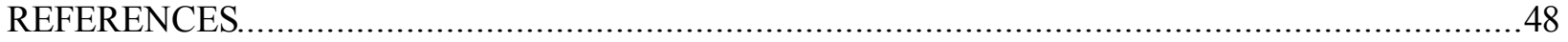




\section{LIST OF TABLES}

Table 1. Participant characteristics stratified by risk category..........................41

Table 2. Bivariate analysis of ceramides by risk factors for cardiovascular disease to assess for possible covariates.................................................42

Table 3. Associations in HEI-20015 and BMI with ceramides and ceramide risk score........43

Supplemental Table. HEI-2015 criteria for scoring................................46 


\section{LIST OF FIGURES}

Figure 1. Serum ceramide concentrations in $\mu \mathrm{mo} / \mathrm{L}$ by BMI categories normal, overweight,

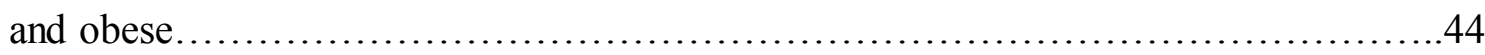

Figure 2. Ceramide risk score by BMI categories normal, overweight, and obese..............45 


\section{LIST OF ABBREVIATIONS}

Acute Coronary Syndrome

ACS

American Heart Association

AHA

Bergen Coronary Angiography Cohort

BCAC

Body Mass Index

BMI

Cardiovascular Disease

CVD

C-Jun N-Terminal Kinases

JNK

Congestive Heart Failure

$\mathrm{CHF}$

Coronary Artery Bypass Graft

$\mathrm{CABG}$

Coronary Artery Disease

$\mathrm{CAD}$

C-Reactive Protein

CRP

Dietary Guidelines for Americans

DGA

Extra Virgin Olive Oil

EVOO

Fat Mass Index

FMI

Fatty Acid Oxidation

FAO

Glucose Transporter Type 4

GLUT4

Hazard Ratio

HR

Healthy Eating Index

HEI

High Density Lipoprotein

HDL

Homeostatic Model Assessment of Insulin Resistance

HOMA-IR

Interleukin 1 beta

IL-1 $\beta$

Low-density Lipoprotein

LDL

Ludwigshafen Risk and Cardiovascular Health

LURIC

Major Adverse Cardiovascular Event

Mediterranean Diet

MACE

Milligrams per Deciliter

MedDiet

Monounsaturated Fatty Acids

$\mathrm{mg} / \mathrm{dL}$

Myocardial Infarction

MUFA

Nano grams per Milliliter

MI

Nano moles per Milliliter

$\mathrm{ng} / \mathrm{mL}$

$\mathrm{nmol} / \mathrm{mL}$

$\mathrm{N}$-arachidoyl-D-erythro-sphingosine

C20:0

N-behenoyl-D-erythro-sphingosine

$\mathrm{C} 22: 0$

$\mathrm{N}$-lignoceroyl sphingosine

$\mathrm{C} 24: 0$

$\mathrm{N}$-nervonoyl sphingosine

C24:1

N-palmitoyl sphingosine

C16:0

$\mathrm{N}$-stearoyl sphingosine

C18:0

Nuclear Factor kappa-light-chain-enhancer of activated B cells

NF-kB

Nutrition Data Systems for Research

Oleic Acid

NDSR

Palmitic Acid

OA

PA

Polyunsaturated Fatty Acids

PUFA

Prevencion con Mediterranean

PREDIMED

Protein Kinase B

Akt

Reactive Oxygen Species

ROS

Receptor for Advanced Glycation End Products

RAGE 
Research Electronic Data Capture

REDCap

Roux-en-Y Gastric Bypass

RYGB

Saturated Fatty Acids

SFA

Serine Palmitoyltransferase

SPt

Special Program University Medicine-Inflammation in Acute Coronary

SPUM-ACS

Syndromes

Sphingomyelinase

SMase

Stable Angina Pectoris

SAP

Toll-like Receptor 4

TLR4

Tumor Necrosis Factor Alpha

TNF- $\alpha$

United States

U.S

Waist Circumference

WC

Waist-Hip Ratio

WHR

West Virginia University

WVU 


\section{INTRODUCTION}

Cardiovascular disease (CVD) continues to be one of the leading causes of death worldwide. ${ }^{1}$ In the United States (U.S.) alone, one in four people die of CVD each year. ${ }^{2}$ Identifying CVD and reducing CVD risk is important to reduce healthcare costs and improve quality of life. Diet has been shown to be a modifiable risk factor for CVD, and evidence-based dietary patterns such as the Mediterranean Diet and adhering to the U.S. Dietary Guidelines for Americans (DGAs) have shown effectiveness in reducing risk of CVD mortality and lowering traditional CVD biomarkers. ${ }^{3,4}$ Traditional biomarkers of CVD risk include low-density lipoprotein (LDL) cholesterol, high density lipoprotein (HDL) cholesterol, and triglycerides. ${ }^{5}$ However, studies looking at CVD mortality have suggested that LDL and triglycerides may not be highly predictive of CVD risk, ${ }^{6,7}$ and there is emerging evidence that another class of lipids, ceramides, may be a better clinical biomarker for predicting CVD risk.

Ceramides are present in all cells and lipoproteins as a type of sphingolipid. Ceramides are biologically active, as they play a role in cell differentiation, apoptosis, cellular stress response, and inflammatory response. ${ }^{8}$ They are synthesized from saturated fats and sphingosine, and this synthesis occurs in all tissues. ${ }^{9}$ In situations such as dyslipidemia and caloric excess, ceramides are synthesized de novo and can accumulate in tissues that are not suited for fat storage, such as the heart. ${ }^{9}$ Levels of circulating ceramides are reflective of tissue accumulation. The current research on ceramides points to associations between circulating ceramides and CVD, along with risk factors for CVD including obesity and insulin resistance. ${ }^{8,10}$

While there are many structurally different species of ceramides present in the body, three specific ceramides were identified as being associated with CVD risk. ${ }^{6,11}$ A ceramide risk score based on $\mathrm{C} 16: 0, \mathrm{C} 18: 0, \mathrm{C} 24: 1$ and their ratios to $\mathrm{C} 24: 0$ has been identified as a new clinical 
biomarker of CVD events and is commercially offered by the Mayo Clinic as of 2016. ${ }^{11,12}$ While the test offers to identify those at risk of CVD, little is known about how to modify ceramide levels. Evidence suggests that these lipids may be modifiable by statin treatment, ${ }^{6}$ gastric bypass, ${ }^{13}$ and aerobic exercise, ${ }^{14}$ but their relationship to diet is largely unknown. Determining if circulating ceramides are associated with diet and body mass index (BMI) is needed to guide the possible development of dietary strategies to lower ceramides and thus, CVD risk.

There are still gaps in the literature centering on ceramides. Across studies, there are variations in the specific ceramide species measured. Furthermore, few studies exist on the associations between diet and ceramides along with other risk factors for CVD, such as obesity. Therefore, the purpose of this thesis is to examine the association of diet quality and BMI with ceramides. We hypothesized that higher diet quality as assessed by the HEI-2015 is associated with lower circulating ceramides and a lower ceramide risk score. We also hypothesized that higher BMI is associated with higher circulating ceramides and a higher ceramide risk score. 


\section{CHAPTER 1: LITERATURE REVIEW}

\section{Ceramide Synthesis}

Ceramide is produced in the body by three main pathways: de novo synthesis, sphingomyelin hydrolysis, and the salvage pathway. ${ }^{8}$ The de novo synthesis pathway is controlled by palmitoyl Co-A and serine. These two combine with the aid of serine palmitoyltransferase (SPt) enzyme to create 3-keto-spinganine which becomes sphinganine, then dihydroxyceramide, and eventually resulting in the end product of ceramide. ${ }^{8}$ The amount of palmitoyl Co-A levels are related to palmitate. Thus, this pathway depends on the availability of saturated fatty acids. ${ }^{8}$ Increased palmitate and serine means there is increased substrate availability for the synthesis of ceramide. ${ }^{8}$ It is suggested that the disruption of ceramide de novo synthesis pathway could have a beneficial impact on metabolism. ${ }^{8}$

The two other ceramide pathways, sphingomyelin hydrolysis and the salvage pathway, require an initial supply of ceramide. ${ }^{8}$ Sphingomyelin hydrolysis requires sphingomyelin, which is the most abundant sphingolipid in mammals. ${ }^{8}$ Sphingomyelinase (SMase) is the enzyme responsible for hydrolyzing sphingomyelin. SMase is activated by pathogens, oxidative stress, and inflammatory signals. Tumor necrosis factor alpha (TNF- $\alpha$ ) and interleukin 1 beta (IL-1 $\beta$ ) both activate SMase and induce ceramide accumulation. ${ }^{8}$

The third pathway of ceramide synthesis, the salvage pathway, produces ceramides via the catabolism of other complex sphingolipids. ${ }^{8}$ However, this is an indirect pathway and not as well researched as the other two pathways. Sphingomyelin and glycosphingolipids are degraded by enzymes to form ceramide. ${ }^{8}$ This occurs within acidic cellular compartments. Ceramide is then degraded to sphingosine and free fatty acids that enter back into the cytosol. Once they are in the cytosol, sphingosine is converted back to ceramide via ceramidase. ${ }^{8}$ 
While ceramide production is controlled by the availability of palmitate and serine, recent studies have shown that tissue ceramide levels are regulated by hormonal signals that control rates of synthesis and degradation. ${ }^{15}$ Inflammatory agonists are increased in obesity, which leads to increased ceramide production. ${ }^{8}$ Additionally, toll-like receptor 4 (TLR4) activation leads to increased production of ceramide biosynthetic enzymes. Saturated fatty acids activate the TLR4 response, but not unsaturated fatty acids. ${ }^{15}$ TLR4 is needed for palmitate-induced insulin resistance and ceramide synthesis, while ceramide synthesis is needed for TLR4-induced insulin resistance. ${ }^{8}$ Palmitate, TLR4, and ceramide work synergistically to cause insulin resistance, which is a risk factor for cardiovascular disease.

Once produced, ceramides are responsible for a number of different mechanisms which alter cellular metabolism. Ceramide inhibits protein kinase B (Akt). ${ }^{8}$ Due to this inhibition, there is decreased insulin signaling, nutrient uptake, glycogen synthesis and protein synthesis. ${ }^{8}$ Akt is also responsible for nutrient catabolism, so there is an upregulation in nutrient storage when it is inhibited. Akt further plays a role in glucose transporter type 4 (GLUT4) signaling. By inhibiting Akt, insulin resistance increases. ${ }^{8}$ Ceramides also inhibit fatty acid oxidation (FAO) in the electron transport chain. ${ }^{10}$ This causes an accumulation of reactive oxygen species (ROS), which can lead to CVD when accumulating in heart muscle and lead to insulin resistance. ${ }^{10}$ Ceramide is also responsible for activating JNK, NF-kB, and the receptor for advanced glycation end products (RAGE), resulting in increased inflammation. ${ }^{10}$ Ceramide has been shown as a downstream mediator of ghrelin and leptin signaling, two hormones involved in hunger and satiety. ${ }^{10}$ Therefore, increased levels of ceramides promote increased food intake and body weight gain, both risk factors for the development of CVD. ${ }^{10}$ 


\section{Serum Ceramides as a Risk Factor for Cardiovascular Disease}

Ceramides and their relationship to CVD risk, along with mortality from CVD, has been explored in a number of studies. As discussed previously, ceramides are present in all lipoproteins, including LDL cholesterol, and play a biochemical role in the progression of atherosclerosis. ${ }^{9}$ When ceramide content is increased within LDL, this promotes infiltration into the vessel wall, contributing to atherosclerosis. ${ }^{9}$ Furthermore, arterial plaque is enriched with ceramides. ${ }^{9}$ Early studies on this topic sought to examine their associations with traditional risk factors of CVD.

Ichi et al. ${ }^{16}$ examined the relationship between ceramide concentration and risk factors of atherosclerosis in human plasma within a sample of 100 Japanese adults who did not have a diagnosis of CVD. In this study, total plasma ceramide was defined as the sum of ceramides C16:0, C18:0, C22:0, C24:0, C24:1, and C24:2. The major ceramides found in human plasma were C24:0 and $\mathrm{C} 24: 1$. The total ceramide concentration showed a significant positive correlation with total cholesterol $(\mathrm{r}=0.651, \mathrm{p}<0.01)$, triglycerides $(\mathrm{r}=0.444, \mathrm{p}<0.01)$, and free fatty acids $(\mathrm{r}=0.266$, $\mathrm{p}<0.01)$. Total plasma ceramide also showed a significant positive correlation with systolic blood pressure $(\mathrm{r}=0.223, \mathrm{p}<0.05)$, but not diastolic blood pressure. Total plasma ceramide level increased drastically at a high level of LDL cholesterol, defined as $170 \mathrm{mg} / \mathrm{dL}$. Additionally, total ceramide did not correlate with HDL cholesterol. This study showed that traditional risk factors for CVD are positively correlated with ceramides in a population with no diagnosis of CVD.

While the subjects in the previous study were healthy individuals, Yu et al. ${ }^{17}$ aimed to document ceramide levels in congestive heart failure (CHF) patients to determine the potential prognostic value. Researchers hypothesized that ceramide levels are upregulated in patients with CHF and associated with the severity of symptoms and mortality. "Ceramide levels" were defined as the sum of ceramides $\mathrm{C} 18: 0, \mathrm{C} 20: 0, \mathrm{C} 22: 0$ and $\mathrm{C} 24: 0$. The population consisted of 423 patients 
with CHF and reduced left ventricular systolic function, along with 104 healthy individuals. After a mean follow-up of 4.4 years, there were 200 deaths total. The optimal threshold value for the prediction of mortality was determined as a ceramide level of $6.05 \mathrm{ng} / \mathrm{mL}$. Thus, a dichotomous variable was created; participants who had plasma ceramide levels above 6.05 were considered the "higher ceramide group". Plasma ceramide level showed a strong predictive value for mortality of CHF both as a continuous and as dichotomous variable, with an adjusted hazard ratio (HR) of 1.31 (95\% CI 1.16-1.47, $\mathrm{p}<0.001)$ for increments of $1 \mathrm{ng} / \mathrm{mL}$ increase as a continuous variable and an adjusted HR of 2.07 (95\% CI 1.53-2.81, $\mathrm{p}<0.001)$ as a dichotomous variable. This study revealed the prognostic value of plasma ceramide levels as predictor of mortality in patients with diagnosed CHF.

These two studies laid the foundation for future research on the relationship between CVD and ceramides. However, inconsistency in measurements of ceramides was seen in both studies, making it difficult to determine which specific ceramides are associated with increased CVD risk.

\section{Ceramide Risk Score}

Many structurally different ceramides are present in humans due to multiple different ceramide synthase enzymes, which each have a unique tissue distribution and fatty acid selectivity. Metabolomics analysis by Tarasov et al. ${ }^{6}$ identified three plasma ceramides as significantly linked to cardiovascular mortality in a cohort with coronary artery stenosis. These ceramides were C16:0, C18:0, and C24:1. This association was independent of traditional risk factors for CVD, including age, smoking status, triglycerides, LDL, and total cholesterol. These ceramides were also normalized (analyzed as a ratio) with $\mathrm{C} 24: 0$, which evidence suggests is a highly abundant plasma ceramide not influenced by disease. ${ }^{6,9}$ Thus, these three individual ceramides and their ratios to C24:0 were narrowed down as predictive markers of CVD risk. 
With this information, Laaksonen et al. ${ }^{11}$ aimed to study the prognostic value of these plasma ceramides as cardiovascular death markers in three independent coronary artery disease cohorts. Each of the three cohorts had a cardiovascular related health condition. The Corogene study cohort consisted of stable coronary artery disease patients who had died within the average follow up of 2.5 years, while the Bergen Coronary Angiography Cohort (BCAC) consisted of 1580 adults with stable coronary artery disease who were followed for 4.6 years with a total of 81 dying of CVD. Finally, participants in the Special Program University Medicine-Inflammation in Acute Coronary Syndromes (SPUM-ACS) cohort all had acute coronary syndromes, and 51 of the 81 cases died from cardiac reasons after one year of follow up.

This study ${ }^{11}$ was the first to develop and use the ceramide risk score as a risk estimation tool for CVD events. The score was calculated by taking the concentrations of the ceramides implicated in the previous study to be predictive of CVD risk (C16:0, C18:0, C24:1, and their ratios to $\mathrm{C} 24: 0)$ and comparing them to the whole study population. ${ }^{6}$ If the ceramide concentration was in the $3^{\text {rd }}$ quartile, the individual received one point, and if the concentration was in the $4^{\text {th }}$ quartile, the individual received two points. Zero points were given if the concentration was in the first or second quartile. Thus, scores ranged from $0-12$, with higher scores corresponding to higher serum ceramide levels. The score is further classified into low (0-2), moderate (3-6), increased (79), and high (10-12) risk categories.

In the Corogene cohort, results showed significant differences between $\mathrm{C} 16: 0, \mathrm{C} 18: 0$, and C24:1 between participants who had a fatal myocardial infarction (MI) during the follow up period and the controls $(17.0 \%, 10.3 \%$, and $11.2 \%$ higher in controls, respectively). Significant odds ratios for coronary death were found in ceramide ratios C16:0/C24:0 (2.83; CI 1.7-4.7), C18:0/24:0 (2.15; CI 1.32-3.48), and C24:1/24:0 (2.72; CI 1.77-4.18). These ratios remained significant after 
adjusting for cholesterol, LDL, triacylglycerol, HDL, and CRP. C16:0 and C18:0 were positively correlated with $\mathrm{C}$-reactive protein $(\mathrm{CRP})(\mathrm{r}=0.28$ and $\mathrm{r}=0.39$, respectively). However, C24:0 was $14.9 \%$ lower in cases who had a fatal MI when compared to the control group, and a negative correlation was seen between C24:0 and CRP $(\mathrm{r}=-0.12)$. In the BECAC cohort, ${ }^{11}$ the same three ceramide ratios as in the Corogene cohort ${ }^{11}$ were significantly higher in the 81 patients who died following a CVD event within 4.6 years of follow up compared to those who did not die during follow up. Statin use did not significantly influence ceramide levels. In the SPUM-ACS cohort, the 51 patients who died following a cardiac event within one year of follow up had significantly higher ceramide ratios compared with those who survived in the follow-up period.

Specifically addressing the ceramide risk score, in the BECAC and SPUM-ACS cohorts, the risk of CVD death increased as scores increased. In stable CAD and ACS patients, a 4.2 and 6.0 fold relative risk increase was observed when comparing the high-to-low risk categories. Thus, evidence from this study suggested that the ceramide risk score may be a predictor of CVD events and mortality.

Other studies have consequently applied the ceramide risk score to their populations to assess CVD and CVD mortality. Havulinna et al. ${ }^{18}$ used the ceramide risk score (C16:0, C18:0, $\mathrm{C} 24: 1$, and their ratios to $\mathrm{C} 24: 0)$ to determine whether ceramides were associated with major adverse cardiovascular events (MACE's) among apparently healthy individuals in the populationbased FINRISK 2002 cohort that included 8,101 individuals of Finland. Within the sample, 813 participants had incident MACE. Increased concentrations of C16:0, C18:0, and C24:1 were observed in individuals who experienced an incident MACE during the follow-up when compared with the asymptomatic individuals $(11.4 \%, 21.3 \%$, and $17.0 \%$, respectively). C18:0 had the strongest association with incident MACE and the highest unadjusted hazard ratio of 1.31. The 
lowest 13-year incident MACE risk (2.6\%) was observed in subjects having both CRP and C18:0 in the lowest concentration quartile. When researchers applied the ceramide risk score to the study population, both MACE and MACE death risk were increased along with the increasing score. A 2.7- and 4.3-fold relative risk increase was observed for MACE and MACE death, respectively, when comparing the high versus the low-risk category, further validating Laaksonen's work. ${ }^{11}$

Similarly, Androedh et al. ${ }^{19}$ investigated the associations of ten previously identified high risk molecular species, specifically those involved in the ceramide risk score, with the occurrence of MACE during a follow up of 4.7 years in patients with $\mathrm{CAD}$ as part of the Ludwigshafen Risk and Cardiovascular Health (LURIC) study. 574 patients were included in the analysis, with 55\% of patients diagnosed with acute coronary syndrome (ACS) and $46 \%$ with stable angina pectoris (SAP). ACS patients had significantly higher plasma concentrations of C16:0, C20:0, C24;0, C24:1, and the ratio C16:0/24:0 in the full cohort, including non-MACE and MACE compared to SAP patients. Additionally, the concentration of C16:0 in ACS patient was significantly higher in the MACE cohort compared to the non-MACE cohort $(\mathrm{p}=0.054)$. In the multivariable analyses between the ceramide concentrations and MACE, after adjustment for cardiac risk factors, clinical presentation, and statin use at baseline, $\mathrm{C} 16: 0$ and $\mathrm{C} 24: 1$ were significantly associated with MACE (HR: $2.14,95 \%$ CI 1.22-3.76, $\mathrm{p}=0.008$ and HR: $1.64,95 \%$ CI 1-2.68, $\mathrm{p}=0.05$, respectively). However, after adjusting for LDL or non-HDL, only C16:0 remained significant. In the univariate analysis, C16:0, C20:0, C24:1, C16:0/24:0, C20:0/24:0 and C24:1/24:0 were significantly associated with death and remained significant after adjusting for cardiac risk factors, indication for coronary angiography, statin use at baseline, and serum LDL. Notably, Androedh et al. did not use the ceramide risk score in their analysis but analyzed the components of the risk score. 
Meeusen et $a .^{20}$ furthered the research on plasma ceramides, specifically the ceramide risk score, and their predictive capacity of MACEs in patients who underwent coronary angiography. The study population were 504 patients who were undergoing clinically indicated coronary angiography at the Mayo Clinic between June 1998 and December 1998. Patients with diabetes and patients who had smoked for more than 50 pack years were not eligible for the study. Incidence of MI, coronary artery bypass surgery (CABG), percutaneous intervention, stroke, and death were evaluated after four years. Participants were again evaluated for death after eighteen years. Plasma ceramides, demographic information, and other traditional CVD biomarkers were measured at baseline. Ceramides measured were: C16:0, C18:0, C24:0, C24:1, and the ratios C16:0/24:0, C18:0/24:0, and C24:1/24:0. The ceramide risk score was also calculated in this study with scores ranging from $0-12$. The risk score was also stratified into four categories $(0-2,3-6,7-9$, and 1012). At baseline, ceramides were directly correlated with total cholesterol $\left(7 \%<\mathrm{R}^{2}<17 \%\right)$, LDL $\left(4 \%<\mathrm{R}^{2}<10 \%\right)$, and triglycerides $\left(7 \%<\mathrm{R}^{2}<18 \%\right)$ and inversely correlated with $\mathrm{HDL}$ $\left(0.2 \%<\mathrm{R}^{2}<3 \%\right)$. The four-year outcomes showed that $\mathrm{C} 16: 0, \mathrm{C} 18: 0$, and $\mathrm{C} 24: 1$ were significantly predictive of MI, stroke, revascularization, and death from any cause. C24:0 was not found to be predictive, but when used in the ratio with $\mathrm{C} 16: 0, \mathrm{C} 18: 0$, and $\mathrm{C} 24: 1$, there was an increased association with those outcomes.

When assessing the ceramide risk score, the score was less than or equal to 6 for $64.6 \%$ of patients. The rate of a cardiovascular event was significantly higher (20\% versus $11 \%)$ among the 65 individuals with a ceramide score greater than or equal to $10(\mathrm{p}=0.04)$. The ceramide risk score was significantly predictive of 4-year outcomes with a fully adjusted per standard deviation HR of 1.58 (95\% CI, 1.22-2.04). Importantly, cardiovascular event rates increased as ceramide score increased in patients who had or did not have CAD at baseline. Kaplan-Meier survival analysis 
showed that risk of major adverse events at any time was significantly greater $(\mathrm{p}<0.0001)$ during 4 years for individuals with increased ceramide risk score. At 18 years, C16:0 and C24:1 were significantly predictive for all-cause death $(\mathrm{p}<0.005)$. The ratios analyzed were significantly predictive $(\mathrm{p}<0.002)$ of all-cause death and remained that way after adjusting for age, sex, BMI, hypertension, smoking, HDL, LDL, triglycerides, serum glucose, and family history of CAD $(p<0.004)$. Furthermore, a ceramide risk score greater than or equal to 10 was associated with $a>2$ fold increase in risk of all-cause death at 18 years compared with patients with a score below 2 $(\mathrm{p}<0.001)$. This study found a strong indication between ceramides and the ceramide risk score in a population with and without CVD.

Studies using the ceramide risk score or simply using the species involved in the ceramide risk score consistently pointed to increased CVD events and mortality in participants with elevated ceramides. Based on the results of these studies, the Mayo Clinic began offering a commercial test for these specific plasma ceramides and categorization by the ceramide risk score for the general public. ${ }^{12}$

\section{Obesity and Ceramides}

Obesity, excessive fat accumulation in adipose tissue, is considered a risk factor for $\mathrm{CVD},{ }^{21}$ and studies have examined the mechanistic link between obesity and ceramide levels. Elevated saturated free fatty acids in circulation promote de novo ceramide synthesis, ${ }^{10}$ and inflammatory cytokines that are elevated in obese individuals activate the Smase enzyme, which regulates the second pathway of ceramide synthesis. ${ }^{10}$ Further, elevated ceramide levels reduce FAO and disrupt the electron transport chain through $\mathrm{C} 18: 0$ targeting mitochondria to induce mitophagy, leading to reduced FAO capacity via mitochondrial depletion. ${ }^{10}$ Therefore, a number of studies have included measures of adiposity and obesity such as BMI and their associations with ceramides. 
In a study examining adiposity measures with plasma ceramides, Mielke et al. ${ }^{22}$ quantified plasma ceramides C16:0, C18:0, C20:0, C22:0, C24:0, C26:0, C22:1 and C24:1 by age and sex in 922 individuals aged 55 and older enrolled in the Baltimore Longitudinal Study of Aging. They found that waist to hip ratio was positively and significantly associated with C18:0, C20:0, C22:0, and C24:1. Higher BMI was also significantly associated with increases in ceramide species C16:0, C18:0, C20:0, C22:0, C22:1, and C24:1. Notably, the mean BMI of their entire population was 26 $\mathrm{kg} / \mathrm{m}^{2}$.

In a study investigating how decreases in fat affect ceramide levels, Huang et al. ${ }^{13}$ examined the effect of Roux-en-Y gastric bypass surgery (RYGB) on weight loss, insulin sensitivity, plasma ceramides, pro-inflammatory markers, and cardiovascular risk factors before surgery and at 3 and 6 months post-surgery. The sample consisted of 13 patients, 10 of which were female, who had undergone RYGB surgery. The plasma ceramides analyzed were C14:0, C16:0, C18:0, C18:1, C20:0, C24:0, and C24:1. The sum of these resulted in the total plasma ceramide level. The predominant ceramides in the plasma of obese patients were C24:1, C24:0 and C16:0. The results showed that total plasma ceramide levels decreased significantly postoperatively, with levels of $9.3+/-0.5 \mathrm{nmol} / \mathrm{ml}$ at baseline vs $7.6+/-0.4$ at 3 months, and $7.3+/-0.3$ at 6 months $(\mathrm{p}<0.05)$. Specifically, gastric bypass resulted in reduced levels of C14:0, C16:0, C20:0, and C24:0. The improvement in insulin sensitivity correlated with the change in total ceramide levels $(\mathrm{r}=0.68, \mathrm{p}=0.02)$ and with plasma TNF- $\alpha(\mathrm{r}=-0.62, \mathrm{p}=0.04)$ at 6 months post-surgery. This study provides evidence that ceramides can be lowered by decreasing adipose tissue.

While not the primary endpoint of many studies, BMI has been assessed in studies analyzing ceramides. Haus et al. saw that C18:0, C20:0, and C24:1 were increased in the plasma of obese subjects with a diagnosis of Type 2 diabetes compared to healthy controls. ${ }^{23}$ In a study 
analyzing ceramide risk score or components of the risk score, Meeusen et al. saw a minimal association with $\mathrm{C} 18: 0$ and $\mathrm{C} 18: 0 / 24: 0\left(\mathrm{R}^{2}<3 \%\right)$ in participants with a clinically ordered coronary angiography. ${ }^{20}$

This evidence presents a link between obesity and ceramides and suggests that weight loss has the potential to decrease ceramide levels. However, BMI was not specifically addressed in the majority of studies looking at ceramides involved in the ceramide risk score, even though obesity is a risk factor for CVD. Only one study looked at the associations between weight loss and ceramides, showing there is still a gap in the literature on how to effectively lower ceramide levels.

\section{Diet and Ceramides}

Diet and its relationship to ceramide levels is a largely unexplored topic, with only a few intervention studies available for review. Ceramides are potentially modifiable by diet, as the substrates of de novo ceramide synthes is depends on the availability of free fatty acids, specifically palmitate. ${ }^{8}$ Palmitate is a saturated fatty acid, with food sources including milk and milk products, red meat, and palm oil. Sources of palm oil include cookies, crackers, and other processed foods that are frequently overconsumed. In addition, hormones that are increased in obesity increase synthesis of ceramides, and dietary changes have been shown to reduce obesity. ${ }^{10}$

Adhering to dietary patterns rather than single nutrients is a target when aiming to reduce chronic disease risk because dietary components are consumed together and are correlated with one another. ${ }^{3,24}$ Specifically, the Healthy Eating Index (HEI), which measures adherence to the U.S DGA's, has been shown to reduce risk of CVD. ${ }^{3}$ Participants with better adherence to this index had over $12 \%$ decreased risk of CVD mortality in a study of 23,502 Americans. ${ }^{3}$ Traditio nal biomarkers of CVD (LDL cholesterol and triglycerides) have also been shown to be decreased with better adherence to the HEI. ${ }^{25}$ As ceramides are now being used as a biomarker of CVD, it is 
important to examine what is known about the association between ceramide levels and diet, a modifiable CVD risk factor.

\section{Overfeeding}

Excess caloric consumption has been linked in a number of studies to increased ceramide levels. When considering how diet can change ceramide levels, Heilbronn et al. ${ }^{26}$ assessed how quickly overfeeding can change the lipidomic profile. Forty healthy individuals were overfed approximately 1,250 kcal per day for 28 days, and the specific ceramides C16:0, C18:0, C20:0, C22:0, C24:0 and C24:1 were analyzed. Results showed that C22:0 and C24:0 increased significantly compared to baseline after the 28 days of overfeeding. This study points to the impact of excessive calorie intake on ceramide levels. This study also did not target a specific macronutrient when overfeeding; rather, a ratio of carbohydrates $(40 \%)$ to protein $(15 \%)$ to fat (45\%) within the 1,250 kcal per day excess was recommended and provided.

In another study on excess calorie consumption, Luukonen et al. ${ }^{27}$ overfed participants to assess the metabolic effects of a hyper caloric diet and tested whether these effects depend on macronutrient composition of the diet. They hypothesized that overconsumption of simple sugars and saturated fat increases availability of saturated fatty acids (SFAs) and thereby ceramide synthesis, and that saturated but not unsaturated fat or simple sugar diets may further increase ceramide synthesis. 38 overweight adult subjects were randomized to one of three intervention groups at baseline: excess energy (1,000 kilocalories) predominately from saturated fat, monounsaturated fat or simple sugars. Participants were prescribed the diet for 3 weeks. Ceramide species measured were C23:0, C24:0, C24:1, C26:0, and C26:1. At baseline, the three groups were comparable with respect to demographics and health characteristics. In the saturated fat group, the sum of all plasma ceramides increased significantly by $49 \%(\mathrm{p}<0.001)$. There were no changes in 
plasma ceramides in the unsaturated fat or the simple sugars group. The increase in total plasma ceramides was significantly higher than in the saturated fat group compared to the unsaturated fat group $(\mathrm{p}<0.05)$ and the simple sugar group $(\mathrm{p}<0.001)$. These findings suggest that excess saturated fat and not unsaturated fat or simple sugars increase ceramides.

Similar to the previous study, Kien et al. ${ }^{28}$ used a randomized crossover trial design to compare a diet high in palmitic acid (PA) to a diet low in PA and high in oleic acid (OA) on insulin sensitivity in women and men. Palmitic acid is a saturated fatty acid and oleic acid is a monounsaturated fatty acid. Total ceramide concentrations were higher during the high PA diet in both the fasting and fed states in both women and men, and nearly every ceramide species measured in serum increased in response to the high PA diet. However, one major limitation of this study is that they did not report specifically which ceramides made up the total concentration.

As the studies by Heilbronn et al. ${ }^{26}$ and Luukonen et al. ${ }^{27}$ showed, 21- 28 days of overfeeding is enough to notice significant increases in the lipidomic and ceramide profile of individuals. The studies by Luukonen et al. ${ }^{27}$ and Kien et al. ${ }^{28}$ point to saturated fat as a target of ceramide increase. However, a lack of uniformity in ceramide measurements was seen between the three studies, making it difficult to make direct comparisons. Regardless, changes in ceramides were seen in short term interventions, making it likely that a dietary intervention lasting three or more weeks could possibly invoke decreases in ceramides. Dietary intervention studies with the goal of decreasing ceramide levels have the potential to provide more information on how a healthy diet could potentially lower ceramides.

\section{Dietary patterns}

Adhering to healthy dietary patterns that target the entire diet have been shown to reduce CVD risk. ${ }^{3}$ For example, the Mediterranean-style eating pattern has been widely recognized as a 
diet to decrease CVD risk. ${ }^{29}$ Characterized as high in fruits, vegetables, whole grains, unsaturated fats, and low in dairy, processed meats, and sweets, ${ }^{29}$ this dietary pattern has been suggested to reduce LDL oxidation and improve HDL cholesterol. Furthermore, this dietary pattern is low in the substrates required for ceramide synthesis. The Nordic Diet is a similar dietary pattern with slight geographical region differences that has also been assessed in regard to ceramides. ${ }^{30}$ The associations between ceramides and dietary patterns have been a target of a few intervention studies.

Wang et al. $^{31}$ used participants from the PREDIMED trial to examine the associations between baseline plasma ceramide concentrations and incident clinical events of CVD, and also to see if the association between baseline plasma ceramide concentrations and incident CVD was modified by Mediterranean Diet (MedDiet) interventions. The sample size of this study was 980 participants, which included 230 incident cases of CVD. Adherence to the Mediterranean diet was assessed via questionnaire, given out by a dietitian at yearly follow-ups. Participants were randomly allocated to one of 3 groups for the intervention: a control group, a MedDiet group supplemented with Extra-Virgin olive oil (EVOO), and a MedDiet group supplemented with nuts. The control group were advised to stick to their typical dietary habits. Compliance to the MedDiet was monitored annually by a 14-item MedDiet screener and by measuring biomarkers of key foods in the MedDiet in random samples of participants. Ceramides measured in the analys is were C16:0, C22:0, C24:0, C24:1, and a weighted sum of these ceramides referred to as a "ceramide score". Notably, these are not the ceramides in the ceramide risk score. The ratios C16:0/24:0, C22:0/24:0, and C24:1/24:0 were also analyzed. At baseline, C24:0 had the highest concentration in plasma, while $\mathrm{C} 16: 0$ had the lowest concentration. C16:0 (HR 2.39, p<0.001), C22:0 (HR 1.91, p=0.003), C24:0 (HR 1.21, p=0.004), and C24:1 (HR 1.73, p=0.11) were positively associated with CVD 
risk at baseline. The ratios $\mathrm{C} 22: 0 / \mathrm{C} 24: 0$ and $\mathrm{C} 24: 1 / \mathrm{C} 24: 0$ were not significantly associated with incidence of CVD. HR for CVD events were higher for participants who had a higher ceramide score and were in the control group $(2.76,95 \% \mathrm{CI}, 1.72-4.44)$, compared to the HR's of participants with a lower ceramide score and randomized to the control group $(1.07,95 \%$ CI 0.64 1.78) and participants with a higher ceramide score and randomized to either of the two active intervention arms (MedDiet with nuts and MedDiet with EVOO). However, one-year changes in ceramide concentration were not significantly different among participants in either of the two intervention groups and those in the control group.

Toledo et al. ${ }^{32}$ also used data from the PREDIMED trial to evaluate three factors: the associations between lipid species and the risk of CVD, a MedDiet intervention and 1 year changes in lipid species, and 1 year changes in lipid species and subsequent CVD. The lipid species C16:0, C22:0, C24:0, and C24:1 were profiled at baseline and after 1 year of the PREDIMED intervention in 983 participants. Intervention groups were the same as described above. Lipid species were collected via blood draw at baseline and after one year of the intervention. Results showed that among other lipid species, ceramide C16:0 and C22:0 were significantly and directly associated with risk of CVD. However, after one year of the intervention, there were no statistically significant differences in any of the ceramides between any of the intervention groups with the control.

While these two studies focused on the Mediterranean diet pattern within the same randomized trial, Lankinen et al. ${ }^{30}$ investigated how a healthy Nordic diet affects fasting plasma lipidomic profile in subjects with metabolic syndrome. One hundred fifty-six participants were randomized to the healthy Nordic diet, which was characterized as a diet high in whole grains, fruits and vegetables, berries, vegetable oil and margarines, fish, low-fat milk products, and low- 
fat meat, or the average Nordic diet, which included low-fiber cereal products, dairy fat-based spreads, regular-fat milk products, and a limited amount of fruits, vegetables, and berries. The ceramides C22:0, 24:0, and 23:0 were measured at baseline, week 12, and end (week 18-24). At week 12 , statistically significant decreases in all ceramides were seen in the healthy Nordic Diet group compared to the control group. However, these differences were no longer significant at the end of the intervention period. Regardless, this study indicated that it is possible to decrease ceramides in as little as 12 weeks of dietary intervention.

In a separate dietary intervention study, Lankinen et al. ${ }^{33}$ investigated how fatty fish or lean fish in the diet affect serum lipidomic profiles in subjects with coronary heart disease. 33 subjects with MI or unstable ischemic attack were participants in the 8-week parallel controlled intervention. 11 were randomized to the fatty fish group (i.e. salmon, rainbow trout, Baltic herring, whitefish, vendace), 12 to the lean fish group (i.e. pike, pike-perch, perch, saithe, and cod), and 10 served as the control. Both fish groups consumed 4 fish meals per week, while the control group was limited to one fish meal per week in addition to pork, beef, and chicken. Ceramide species measured were $\mathrm{C} 23: 0$ and $\mathrm{C} 24: 1$. Notably, there were not significant differences between the three groups at baseline. The results showed that ceramides C23:0 and C24:1 decreased significantly in the fatty fish group compared to the control group at the end of the intervention., with authors concluding that increased intake of long chain n-3 fatty acids diminished ceramides in the fatty fish group.

While it appears that dietary interventions with healthful eating patterns have the ability to decrease ceramides, studies on this topic lack consistency among ceramides analyzed. Further, after one year, the MedDiet intervention in the PREDIMED trial did not change plasma ceramide levels. ${ }^{31,32}$ Adhering to a Nordic Diet showed the ability to decrease ceramides after 12 weeks, as 
did fatty fish intake. Only two dietary patterns (the Mediterranean and Nordic diets) were assessed, which were specific to the regions in the study. In order to understand the impact that diet has on ceramides, more studies need to be done assessing uniform ceramides and looking at other dietary patterns.

\section{Conclusion}

After analyzing the published literature on ceramides and their relationship to CVD, the evidence suggests that there is a link between elevated ceramides and higher risk of CVD and greater mortality from CVD. Specifically, C16:0, C18:0, C24:1, and their ratios to C24:0, showed increased risk of CVD events or mortality in five studies. ${ }^{6,11,18-20}$ In these studies, C24:0 was negatively associated with CVD risk. Further, a higher ceramide risk score showed increased risk of CVD events or mortality in multiple studies. ${ }^{11,18,20}$ Obesity, a risk factor for CVD, also consistently showed a relationship with elevated ceramides. ${ }^{13,22,23}$ Diet, considered a modifiable risk factor for CVD, has been explored in regard to ceramides. Overfeeding studies showed that increases in ceramides were present after only 3-4 weeks of excess calorie consumption, ${ }^{26-28}$ and specifically saturated fat in two studies. ${ }^{27,28}$ Dietary intervention studies with the aim of decreasing ceramides are inconsistent and sparse. Only two dietary patterns- the Mediterranean diet and the Nordic Diet- were analyzed for associations with ceramides in Spain and Finland, respectively. ${ }^{30-32}$ No studies looked at adherence to other dietary patterns that have been shown to reduce CVD risk and obesity, such as the U.S. DGAs. Therefore, analyzing specific dietary patterns that have been associated with reduced CVD risk and reduced obesity, such as the HEI, would be the logical next steps in order to close the knowledge gap between ceramides, diet, and CVD. 


\section{REFERENCES}

1. WHO $\mid$ Cardiovascular diseases (CVDs). WHO. http://www.who.int/cardiovascular_diseases/en/. Accessed November 1, 2018.

2. Centers for Disease Control and Prevention. Heart Disease Facts \& Statistics $\mid$ cdc.gov. https:/www.cdc.gov/heartdisease/facts.htm. Published October 9, 2018. Accessed October 24, 2018.

3. Reedy J, Krebs-Smith SM, Miller PE, et al. Higher diet quality is associated with decreased risk of all-cause, cardiovascular disease, and cancer mortality among older adults. J Nutr. 2014;144(6):881-889. doi:10.3945/jn.113.189407

4. Recommended Dietary Pattern to Achieve Adherence to the American Heart Association/American College of Cardiology (AHA/ACC) Guidelines: A Scientific Statement From the American Heart Association | Circulation. https://www.ahajournals.org/doi/full/10.1161/CIR.0000000000000462. Accessed October $16,2018$.

5. Vasan Ramachandran S. Biomarkers of Cardiovascular Disease. Circulation. 2006;113(19):2335-2362. doi:10.1161/CIRCULATIONAHA.104.482570

6. Tarasov K, Ekroos K, Suoniemi M, et al. Molecular lipids identify cardiovascular risk and are efficiently lowered by simvastatin and PCSK9 deficiency. JClin Endocrinol Metab. 2014;99(1):E45-52. doi:10.1210/jc.2013-2559

7. Sachdeva A, Cannon CP, Deedwania PC, et al. Lipid levels in patients hospitalized with coronary artery disease: an analysis of 136,905 hospitalizations in Get With The Guidelines. Am Heart J. 2009;157(1):111-117.e2. doi:10.1016/j.ahj.2008.08.010

8. Bikman BT, Summers SA. Ceramides as modulators of cellular and whole-body metabolism. J Clin Invest. 2011;121(11):4222-4230. doi:10.1172/JCI57144

9. Meeusen JW, Donato LJ, Jaffe AS. Lipid Biomarkers for Risk Assessment in Acute Coronary Syndromes. Curr Cardiol Rep. 2017;19(6):48. doi:10.1007/s11886-017-0863-9

10. Fucho R, Casals N, Serra D, Herrero L. Ceramides and mitochondrial fatty acid oxidation in obesity. FASEB J. 2017;31(4):1263-1272. doi:10.1096/fj.201601156R

11. Laaksonen R, Ekroos K, Sysi-Aho M, et al. Plasma ceramides predict cardiovascular death in patients with stable coronary artery disease and acute coronary syndromes beyond LDLcholesterol. Eur Heart J. 2016;37(25):1967-1976. doi:10.1093/eurheartj/ehw148

12. Mayo Clinic. Plasma Ceramides: A Novel Biomarker of Unstable Atherosclerotic Cardiovascular Disease.; :4. https://cdn.prodcarehubs.net/n1/96e99366 cea7b0de/uploads/2016/07/ceramides-brochure-final-0616.pdf. 
13. Huang H, Kasumov T, Gatmaitan P, et al. Gastric bypass surgery reduces plasma ceramide subspecies and improves insulin sensitivity in severely obese patients. Obesity (Silver Spring). 2011;19(11):2235-2240. doi:10.1038/oby.2011.107

14. Bergman BC, Brozinick JT, Strauss A, et al. Serum sphingolipids: relationships to insulin sensitivity and changes with exercise in humans. Am J Physiol Endocrinol Metab. 2015;309(4):E398-408. doi:10.1152/ajpendo.00134.2015

15. Holland WL, Bikman BT, Wang L-P, et al. Lipid-induced insulin resistance mediated by the proinflammatory receptor TLR4 requires saturated fatty acid-induced ceramide biosynthesis in mice. J Clin Invest. 2011;121(5):1858-1870. doi:10.1172/JCI43378

16. Ichi I, Nakahara K, Miyashita Y, et al. Association of ceramides in human plasma with risk factors of atherosclerosis. Lipids. 2006;41(9):859-863. doi:10.1007/s11745-006-5041-6

17. Yu J, Pan W, Shi R, et al. Ceramide is upregulated and associated with mortality in patients with chronic heart failure. Can J Cardiol. 2015;31(3):357-363. doi:10.1016/j.cjca.2014.12.007

18. Havulinna AS, Sysi-Aho M, Hilvo M, et al. Circulating Ceramides Predict Cardiovascular Outcomes in the Population-Based FINRISK 2002 Cohort. Arterioscler Thromb Vasc Biol. 2016;36(12):2424-2430. doi:10.1161/ATVBAHA.116.307497

19. Anroedh SS, Hilvo M, Akkerhuis KM, et al. Plasma concentrations of molecular lipid species predict long-term clinical outcome in coronary artery disease patients. J Lipid Res. June 2018. doi:10.1194/j1r.P081281

20. Meeusen JW, Donato LJ, Bryant SC, Baudhuin LM, Berger PB, Jaffe AS. Plasma Ceramides: A Novel Predictor of Major Adverse Cardiovascular Events After Coronary Angiography. Arterioscler Thromb Vasc Biol. June 2018. doi:10.1161/ATVBAHA.118.311199

21. Poirier Paul, Giles Thomas D., Bray George A., et al. Obesity and Cardiovascular Disease: Pathophysiology, Evaluation, and Effect of Weight Loss. Circulation. 2006;113(6):898918. doi:10.1161/CIRCULATIONAHA.106.171016

22. Mielke MM, Bandaru VVR, Han D, et al. Demographic and clinical variables affecting mid- to late-life trajectories of plasma ceramide and dihydroceramide species. Aging Cell. 2015;14(6):1014-1023. doi:10.1111/acel.12369

23. Haus JM, Kashyap SR, Kasumov T, et al. Plasma ceramides are elevated in obese subjects with type 2 diabetes and correlate with the severity of insulin resistance. Diabetes. 2009;58(2):337-343. doi:10.2337/db08-1228

24. Ocké MC. Evaluation of methodologies for assessing the overall diet: dietary quality scores and dietary pattern analysis. Proc Nutr Soc. 2013;72(2):191-199. doi:10.1017/S0029665113000013 
25. Wu P-Y, Huang C-L, Lei W-S, Yang S-H. Alternative health eating index and the Dietary Guidelines from American Diabetes Association both may reduce the risk of cardiovascular disease in type 2 diabetes patients. J Hum Nutr Diet. 2016;29(3):363-373.

doi:10.1111/jhn.12317

26. Heilbronn LK, Coster ACF, Campbell LV, et al. The effect of short-term overfeeding on serum lipids in healthy humans. Obesity (Silver Spring). 2013;21(12):E649-659. doi:10.1002/oby.20508

27. Luukkonen PK, Sädevirta S, Zhou Y, et al. Saturated Fat Is More Metabolically Harmful for the Human Liver Than Unsaturated Fat or Simple Sugars. Diabetes Care. 2018;41(8):1732-1739. doi:10.2337/dc18-0071

28. Kien CL, Bunn JY, Poynter ME, et al. A lipidomics analysis of the relationship between dietary fatty acid composition and insulin sensitivity in young adults. Diabetes. 2013;62(4):1054-1063. doi:10.2337/db12-0363

29. Estruch R, Ros E, Salas-Salvadó J, et al. Primary Prevention of Cardiovascular Disease with a Mediterranean Diet. New England Journal of Medicine. 2013;368(14):1279-1290. doi:10.1056/NEJMoa1200303

30. Lankinen M, Schwab U, Kolehmainen M, et al. A Healthy Nordic Diet Alters the Plasma Lipidomic Profile in Adults with Features of Metabolic Syndrome in a Multicenter Randomized Dietary Intervention. J Nutr. 2016;146(4):662-672. doi:10.3945/jn.115.220459

31. Wang DD, Toledo E, Hruby A, et al. Plasma Ceramides, Mediterranean Diet, and Incident Cardiovascular Disease in the PREDIMED Trial. Circulation. March 2017:CIRCULATIONAHA.116.024261. doi:10.1161/CIRCULATIONAHA.116.024261

32. Toledo E, Wang DD, Ruiz-Canela M, et al. Plasma lipidomic profiles and cardiovascular events in a randomized intervention trial with the Mediterranean diet. Am J Clin Nutr. 2017;106(4):973-983. doi:10.3945/ajen.116.151159

33. Lankinen M, Schwab U, Erkkilä A, et al. Fatty fish intake decreases lipids related to inflammation and insulin signaling--a lipidomics approach. PLoS ONE. 2009;4(4):e5258. doi:10.1371/journal.pone.0005258 


\section{CHAPTER 2: Association of Diet Quality and Body Mass Index with Serum Ceramides in Middle-aged Adults with at Least One Risk Factor for Cardiovascular Disease}

Margaret A. Drazba ${ }^{1}$, Melissa Ventura-Marra ${ }^{*}$, RDN, Ida Holásková ${ }^{2}$, Nadine Sayhoun ${ }^{3}$

${ }^{1}$ Division of Animal and Nutritional Sciences, West Virginia University, Morgantown, West Virginia;

${ }^{2}$ Office of Statistics, West Virginia Agriculture and Forestry Experiment Station, Morgantown, West Virginia

${ }^{3}$ Department of Nutrition and Food Science, University of Maryland, College Park, Maryland.

\section{*Corresponding Author}

Melissa Ventura-Marra, PhD, RDN

2204 Agricultural Sciences Building, PO Box 6108

Morgantown, WV 26506

Tel: 304-293-2690

Email: Melissa.Marra@mail.wvu.edu

Conflict of Interest: The authors declare they have no conflicts of interest related to the study.

Funding Disclosure: This work was supported by the WVU Provost's Office, Health Disparities Mountains of Excellence flash fund award; the USDA National Institute of Food and Agriculture (NIFA) Hatch/Multi-State project 1009924 (WVU) and the West Virginia Agricultural and Forestry Experiment Station. Software was provided by the West Virginia Clinical and Translational Science Institute (National Institute of General Medical Sciences of the National Institutes of Health under Award Number 2U54GM104942-02). The content is solely the responsibility of the authors and does not necessarily represent the official views of the National Institutes of Health. 


\begin{abstract}
Ceramides are a class of lipids that have been implicated in the pathogenesis of cardiovascular disease (CVD). A commercial test of ceramides has been offered by the Mayo Clinic to assess CVD risk via a ceramide risk score, but it is unclear how to lower the score. Previous literature shows that ceramides can be modified by statin use, weight loss, and exercise, but the relationship to diet and body mass index (BMI), both modifiable risk factors for CVD, is largely unknown. The purpose of this cross-sectional study was to determine the association of the Healthy Eating Index 2015 (HEI-2015) and BMI with circulating ceramides in middle-aged adults ( $n=96)$. Dietary intake was assessed using three 24-hour recalls and Nutrition Data System for Research (NDSR) software. Diet quality was estimated using the HEI-2015. Serum ceramide concentrations were determined by quantitative liquid chromatography mass spectrometry (LC/MS). A ceramide risk score was calculated based on published metrics. After adjusting for confounding cardiovascular risk factors, BMI $\left(\mathrm{kg} / \mathrm{m}^{2}\right)$ was positively associated with C18:0 $\left(\mathrm{R}^{2}=0.31, \mathrm{p}<0.0001\right), \mathrm{C} 18: 0 / \mathrm{C} 24: 0\left(\mathrm{R}^{2}=0.30, \mathrm{p}<0.0001\right)$ and the ceramide risk score $\left(\mathrm{R}^{2}=0.11\right.$ $\mathrm{p}<0.009)$. HEI-2015 was inversely associated with $\mathrm{C} 22: 0\left(\mathrm{R}^{2}=0.42, \mathrm{p}=0.02\right)$. These findings suggest that BMI is more predictive of ceramide concentration than diet quality as measured by the HEI-2015 in this sample. Studies to determine if reduction in weight status results in lower ceramide risk scores in humans are needed.
\end{abstract}




\section{Introduction}

Cardiovascular disease (CVD) is one of the leading causes of death worldwide. ${ }^{1}$ In the United States (U.S.), one in four people die of CVD each year. ${ }^{2}$ Traditional risk markers of CVD include elevated LDL cholesterol, elevated triglycerides, decreased HDL, and elevated blood pressure. $^{3}$ However, studies assessing CVD mortality have shown that LDL may not always identify populations at high CVD risk. In a study assessing LDL levels of over 137,000 patients hospitalized for a cardiovascular event, over half of the patients had LDL within a normal range, ${ }^{4}$ suggesting that those at CVD risk are not being identified before the disease progresses to a cardiovascular event. Recent advances in mass spectroscopy have allowed for the measuring of several new small-molecule metabolites that may be prognostic markers for $\mathrm{CVD},{ }^{5}$ one of which are ceramides.

Ceramides are a class of lipids that play a role in cell differentiation, apoptosis, and inflammation, ${ }^{6}$ which contribute to the progression of chronic disease, specifically atheroscleros is development. $^{7}$ Due to six different ceramidase enzymes, there are many structurally unique ceramides in the body with differing functions. ${ }^{8-11}$ In 2016, the Mayo Clinic began offering a commercial test of the ceramides specifically associated with increased CVD risk. ${ }^{12}$ Patients can now have their ceramides measured and a "risk score" determined. The risk score is based on three specific ceramides that have been associated with CVD mortality, and their ratios to C24:0, a ceramide not shown to be associated with CVD. ${ }^{8,13-15}$ Participants classified as "higher risk" are four times more likely to suffer a CVD event than those at lower risk, ${ }^{13,14}$ but it is not clear how to lower the ceramide risk score and thereby reduce CVD risk. Studies have shown that ceramides can be modified through exercise, ${ }^{16}$ statin use,${ }^{8}$ and weight loss. ${ }^{17}$ Only one study has shown that ceramides can be lowered by weight loss, and this was achieved through gastric bypass surgery, ${ }^{17}$ suggesting a link between ceramides and obesity. 
The relationship between diet, a modifiable risk factor for CVD, and ceramides is largely unknown. Short-term intervention studies have shown that caloric excess and increased saturated fat intake increase ceramide levels. ${ }^{18-20}$ In one study, an intervention with the Nordic diet reduced ceramides, specifically C22:0. ${ }^{21}$ In another study, participants who adhered to the Mediterranean dietary pattern had reduced CVD risk compared to a control group, but no changes in ceramides were observed after one year. ${ }^{22} \mathrm{LDL}$, the most widely used method for assessing CVD risk and therefore most commonly studied, has been shown to be reduced in people who adhered to the U.S. Dietary Guidelines for Americans (DGAs) as assessed by the HEI-2010. ${ }^{23}$ Another study showed that adhering to the HEI-2010 reduced CVD mortality. ${ }^{24}$ However, no studies have assessed how adherence to the updated HEI-2015 impacts ceramides or the ceramide risk score. A lack of consistency among specific ceramide species studied and inconsistent results with diet has made it difficult to understand the factors that can lower the ceramide risk score. Therefore, determining if circulating ceramides involved in the risk score are associated with modifiable risk factors for CVD, such as diet and body mass index (BMI), a measure of obesity, through crosssectional studies is the first step in order to guide the possible development of dietary strategies to lower ceramides and thus, CVD risk.

The purpose of this study was to determine if the HEI-2015, a measure of adherence to the U.S DGAs, and BMI were associated with serum ceramides in a middle-aged population with at least one risk factor for CVD. It was hypothesized that higher diet quality as measured by the HEI2015 would be inversely related to ceramides and the ceramide risk score. In addition, it was hypothesized that higher BMI would be associated with higher ceramides and ceramide risk score.

\section{Methods}

Study Sample and Design 
In this cross-sectional study, data were analyzed from 96 participants 45 to 64 years of age who took part in a diet and cardiovascular risk assessment study. Study protocol (1507753607) was approved by West Virginia University (WVU) Institutional Review Board. All participants provided informed consent prior to participation and received a $\$ 100$ gift card upon completion of the study. Participants were recruited from two counties in north central West Virginia by word-of-mouth and community advertising. Exclusion criteria included: current smokers, current diagnosis of cancer or kidney, heart or liver disease; surgery 6 months prior; anti-inflammatory or anti-coagulant medication use; major diet/appetite change 3 months prior or abuse of alcohol or other substances.

The study consisted of three modes of data collection: a series of three telephone interviews to assess dietary intake; an online survey administered using REDCap (Research Electronic Data Capture), a secure web-based application designed to support data capture for research studies and hosted at WVU; ${ }^{25}$ and an in-person health assessment. At the in-person session, anthropometric and blood pressure measurements were taken by research staff, and a fasting venous blood draw was performed by a trained phlebotomist.

\section{Demographic and Health-related Data}

Demographic data (i.e. age and sex) and smoking history were self-reported via the online survey. Participants provided their health and medication history at the in-person visit. A portion of the blood collected at the in-person visit was analyzed by WVU Hospital lab [i.e. LDL cholesterol, HDL cholesterol, non-HDL cholesterol, triglycerides, glucose, insulin, C-reactive protein (CRP) and fibrinogen]. Insulin sensitivity was assessed by the homeostatic model assessment of insulin resistance (HOMA-IR) using glucose and insulin values. Participants were classified as having a health condition (hypertension, dyslipidemia, diabetes, or metabolic 
syndrome) if any one of the three criteria were met: 1) they self-reported ever having been diagnosed with the condition by a health care provider, 2) they reported taking a medication used to treat the condition, or 3) their researcher-obtained laboratory values or blood pressure (BP) measurements met standard diagnostic cut-off values (pre-diabetes or diabetes if fasting plasma glucose $>100 \mathrm{mg} / \mathrm{dL} ; 26$ dyslipidemia if $\mathrm{LDL} \geq 100 \mathrm{mg} / \mathrm{dL}$ or triglycerides $\geq 150 \mathrm{mg} / \mathrm{dL}$; hypertension if systolic BP was $>120$ or diastolic was BP was $>80 \mathrm{~mm} \mathrm{Hg},{ }^{27}$ and the metabolic syndrome if they met 3 of the 5 factors defined by the AHA's diagnostic criteria). ${ }^{28}$ BP was measured using the Omron HEM-907XL Intellisense ${ }^{\circledR}$ Automatic Oscillatory Digital Blood Pressure automatic inflation sphygmomanometer (Omron Health Care, Lake Forest, IL). ${ }^{29}$ A single researcher performed all blood pressure measurements. Arm circumference was measured to the nearest $0.1 \mathrm{~cm}$ in order to determine cuff size based on manufacturer recommendations. With the participant in a seated upright position and after an initial rest of 5 minutes, the machine took three blood pressure measurements at 30 -second intervals; the average reading was used for analysis. ${ }^{30}$

Anthropometric and body composition measurements were taken using standardized protocols, with participants fasted, lightly clothed and without shoes. Measurements were recorded in duplicate and averages were used for analysis. Height $(\mathrm{cm})$ was measured using the Seca 274 digital mobile stadiometer (Seca, Hamburg, Germany). Weight $(\mathrm{kg})$ and fat mass index (FMI) $\left(\mathrm{kg} / \mathrm{m}^{2}\right)$ were measured using the Seca medical Bioelectrical Composition Analyzer (mBCA) 514 (Seca, Hamburg, Germany). BMI was calculated as weight $(\mathrm{kg}) /$ height $\left(\mathrm{m}^{2}\right)$ and was classified using World Health Organization classifications. ${ }^{31}$ Waist and hip circumference (cm) were measured using a Gulick II Tape Measure. Waist circumference (WC) was measured at the iliac crest; values $>102 \mathrm{~cm}$ for men and $>88 \mathrm{~cm}$ for women were classified 'at risk'. ${ }^{31}$ Hip 
circumference was measured at the maximum point of protuberance of the buttocks. Waist-hip ratio (WHR) was calculated as waist circumference $(\mathrm{cm}) /$ hip circumference $(\mathrm{cm})$; values were classified as 'at risk' if WHR was $\geq 0.90 \mathrm{~cm}$ for men and $\geq 0.85 \mathrm{~cm}$ for women.

\section{Diet Quality}

Dietary intake data was collected and assessed using three 24-hour dietary recalls and Nutrition Data Systems for Research (NDSR) software version 15 (2015) developed by the Nutrition Coordinating Center, University of Minnesota, Minneapolis, MN. Self-reported dietary intake was obtained via telephone interview by trained research personnel using the NDSR fourpass method on non-consecutive days (one weekend and two weekdays). Food and nutrient data was transformed a priori into the HEI-2015. The minimum and maximum scores by food categories of the HEI-2015 are provided in Supplemental Table 1.

The HEI-2015 was created by the U.S. Department of Agriculture and the National Cancer Institute ${ }^{32}$ and measures adherence to the 2015-2020 Dietary Guidelines for Americans (DGAs). ${ }^{33}$ The HEI-2015 score includes 13 components (nine adequacies and four moderation) and uses a density approach to set serving standards. To calculate the HEI-2015, NDSR output data was transformed into HEI component variables using NDSR's unpublished guide. ${ }^{34}$ Food group servings (total fruits, whole fruits, total vegetables, greens and beans, whole grains, dairy, total protein foods, seafood and plant proteins, and refined grains) were converted to total servings per 1,000 kilocalories and sodium intake to mg per 1,000 kilocalories. A ratio of polyunsaturated fatty acids (PUFAs) and monounsaturated fatty acids (MUFAs) to saturated fatty acids (SFAs) was generated by dividing the sum of PUFAs and MUFAs by SFAs. Added sugars and saturated fats were assessed as percent of kilocalories. Once the NDSR components were in consistent units with the HEI metric, the simple HEI scoring algorithm method for 
multiple days of intake data was applied. ${ }^{32}$ Component scores were summed for a total score ranging from 0-100, with higher scores indicating better adherence to the 2015 U.S. DGAs.

\section{Serum Ceramides}

Lipidomics analysis was conducted by the WVU Metabolomics Core using liquid chromatography mass spectrometry (LC-MS). Serum samples were extracted using a modified Bligh and Dyer procedure using C12:0-ceramide as an internal standard (Avanti Polar Lipids, Alabaster, AL) using previously described methods. ${ }^{35,36}$ Following liquid-liquid extraction, the organic layer was dried under nitrogen gas (Organomation Associates Inc., Berlin, MA) and resuspended in pure methanol before analysis. Ceramides were separated by gradient elution using UHPLC (ExionLC AD, SCIEX, Framingham, MA) on a C18 reverse-phase column (Phenomenex, Torrence, CA). Ceramides were detected using ESI-MS/MS as previously described (QTRAP 5500, SCIEX). ${ }^{37}$ Ionspray source voltage was 5,000 V at a temperature of $500^{\circ} \mathrm{C}$. Nebulizer, heater, curtain, and collision gas pressures were maintained at $70,60,28$, and 9 psi, respectively. Ceramide ionization parameters were optimized individually, ranging from declustering potential $30-50 \mathrm{~V}$, entrance potential $10-15 \mathrm{~V}$, collision energy $32-37 \mathrm{~V}$, and collision cell exit potential $13-17 \mathrm{~V}$. Ceramides were measured by multiple reaction monitoring of the protonated molecular ion with transition ion of $264.2 \mathrm{~m} / \mathrm{z}$. Eleven-point calibration curves $(0.1 \mathrm{ng} / \mathrm{mL}$ to $10 \mu \mathrm{g} / \mathrm{mL})$ were constructed by plotting the area under the curve for $\mathrm{C} 16: 0-, \mathrm{C} 18: 0-$ , C22:0-, and C24:0-ceramide (Avanti Polar Lipids). Fluctuations in extraction and ionization efficiencies were controlled by normalizing to the $\mathrm{C} 12: 0$-ceramide response and samples were re-run if the internal standard response deviated more than $20 \%$ from its median value.

Concentrations were determined by curve fitting the identified ceramide species based on acylchain length. Standards were injected in duplicate to ensure similar response (the overall mean 
$\mathrm{CV}$ was $\left.12.08 \% ; \mathrm{R}^{2} \geq 0.985\right)$. Instrument control and quantitation were performed using Analyst 1.6.3 and MultiQuant 3.0.2 software, respectively (SCIEX).

Ceramides used in this analysis included those identified in the literature as being related to diet or CVD risk: the six used by the Mayo Clinic's ceramide risk score (C16:0, C18:0, and C24:1 and their ratios to $\mathrm{C} 24: 0)$ and two additional ceramides (C20:0 and C22:0). A ceramide risk score was calculated by assigning a value $0-2$ to each of the six ceramide components that make up the risk score based on published cut-points. ${ }^{13,14}$ The six component scores were summed for a total ceramide risk score ranging from $0-12$, with higher scores indicating higher risk of adverse cardiovascular events. The scores were categorized into one of four risk groups [no risk (0-2), moderate risk (3-6), increased risk (7-9) and higher risk (10-12)]. For this study, score were collapsed into two categories (no risk and moderate risk) as only three participants had scores in the increased risk category and none were in the high risk category.

\section{Statistical Analysis}

Demographic and health-related data were reported as means and standard errors of the mean (SEM) or frequency with percentage, when appropriate. Ceramides and blood biomarkers [i.e, cholesterol, triglycerides, glucose] for CVD were log-transformed to achieve normal distribution. Medians and interquartile ranges were reported for non-normally distributed variables. To assess differences between ceramide risk score categories for all demographic and clinical characteristics, Student's t-test or Chi-square tests were performed, when appropriate. Bivariate analysis was performed to assess the relationship between potential confounding variables (demographics, health conditions, CVD biomarkers, inflammatory markers, and lifestyle factors) and each individual ceramide and the ceramide risk score. The BenjaminiHochberg procedure was performed in order to control for excessive Type I error due to multiple 
analyses, with a false discovery rate set to $10 \%$. The variables that remained significant after the Benjamini-Hochberg procedure were entered into a stepwise regression model, with alpha-toenter set at 0.15 and alpha-to-remove set at 0.15 . This enabled us to estimate the effect of multiple variables on ceramides and ceramide risk score. The variables that remained significant were included in the adjusted model. Multiple linear regression models were done to assess the hypothesized relationship between the HEI-2015 diet quality score and BMI on a continuous scale with individual ceramides along with the ceramide risk score. These models enabled us to assess if the HEI-2015 or BMI were associated with ceramide levels, when adjusted for other significant clinical, demographic or lifestyle characteristics. BMI was further analyzed categorically against each individual ceramide and the ceramide risk score using one-way ANOVA and Tukey's HSD for comparison of BMI categories (normal, overweight, and obese).

All data analyses were performed using JMP and SAS software (JMP®, Version Pro 12.2, SAS Institute Inc., Cary, NC, Copyright C2015; SAS®, Version 9.4, SAS Institute Inc., Cary, NC, Copyright (C2002-2012). Significance criterion alpha for all tests was 0.05.

\section{Results}

\section{Subject Characteristics by Ceramide Risk Category}

Overall, the mean age of the sample was $54 \pm 4.7$ years old and most were non-Hispanic white (95.8\%), college educated (57.3\%) and had annual household incomes greater than $\$ 50,000(67.4 \%)$. The average BMI was $30.85 \pm 7.23$ and just over half $(51.1 \%)$ were classified as "obese". Over half were women $(57.3 \%)$. When participants were categorized by ceramide risk scores classification, $58.3 \%$ were in the low risk group, $38.5 \%$ were at moderate risk, $3.1 \%$ at 'increased risk' and $0 \%$ were at 'high' risk. The moderate and increased-risk groups were collapsed for descriptive purposes. Sociodemographic and health-related characteristics by ceramide risk category are presented in Table 1. Participants classified at 'moderate risk' 
compared to 'low-risk' had significantly higher BMI and FMI, elevated fibrinogen levels (35.0\% compared to $14.3 \%, \mathrm{p}=0.02)$, higher rates of metabolic syndrome $(52.5 \%$ vs $28.6 \%, \mathrm{p}=0.02)$, and had formerly smoked $(62.5 \%$ compared to $41.1 \%, \mathrm{p}=0.04)$.

\section{Relationship between Covariates and Serum Ceramide}

Table 2 depicts the results of bivariate analysis of CVD risk factors by individual ceramides and the ceramide risk score. Age, gender, income, statin use, and hypertension were not significantly associated with any of the ceramides or the ceramide risk score. All of the adiposity measures (i.e., WC, WHR, and FMI); several laboratory values (LDL, HDL, non-HDL, triglycerides, glucose, insulin, HOMA-IR, fibrinogen and CPR); having a diagnosis of diabetes and metabolic syndrome, and being a former smoker were each significantly associated with at least one ceramide or the ceramide risk score. However, after stepwise regression, only FMI, LDL, HDL, non-HDL, glucose, and fibrinogen remained significantly associated with individual ceramides or the ceramide risk score, and therefore were the only covariates included in the adjusted models.

\section{Relationship of Diet Quality and BMI with Serum Ceramides}

Table 3 shows associations between the HEI-2015 and BMI with ceramides and the ceramide risk score. Ceramides not listed in the table, C16:0, C24:0, and C24:1/24:0, were not associated with any of the outcome variables. In unadjusted models, diet quality measured by HEI-2015 was inversely associated with C22:0. BMI was associated with C18:0, C16:0/24:0, C18:0/C24:0 and the ceramide risk score. After adjusting each ceramide for its specific confounding variables, the only ceramide related to diet quality was $\mathrm{C} 22: 0$, which remained inversely associated with HEI-2015. BMI remained positively associated with C18:0, $\mathrm{C} 18: 0 / \mathrm{C} 24: 0$ and the ceramide risk score after adjusting for covariates. 
Figure 1 depicts ceramide values by BMI category. Of the 96 participants, 22 (22.9\%) were classified as normal, $25(26.0 \%)$ overweight, and $49(51.0 \%)$ obese. The ceramide values were significantly higher in participants classified as obese compared to those classified as normal weight for four of the six ceramide values used in the ceramide risk score $(\mathrm{C} 16: 0, \mathrm{C} 18: 0$, C18:0/24:0, C24:1/24:0) and additionally, C20:0. Four of the six ceramides values in the risk score (C16:0, C18:0, C16:0/24:0 and C18:0/24:0) were significantly higher in participants classified as overweight compared to normal weight. Participants classified as obese had significantly higher C18:0 than participants classified as overweight. Figure 1 shows the ceramide risk score by BMI category. The risk score increased by BMI category and was significantly higher between participants classified as normal weight versus obese $(p=0.001)$.

\section{Discussion}

In the present cross-sectional study investigating the association between diet quality, BMI, and ceramides, we found that BMI was positively associated with C18:0, C18:0/24:0, and the ceramide risk score. We did not see an association between the HEI-2015 and the ceramides involved in the ceramide risk score or the ceramide risk score itself, but we found that adherence to the HEI-2015 was inversely associated with C22:0, a ceramide associated in the literature with diet but not included in the ceramide risk score.

\section{BMI}

We found that BMI measured on a continuous scale was positively associated with C18:0, C18:0/24:0, and the ceramide risk score, after adjusting for confounding variables. These findings are similar to previous studies that analyzed ceramides and BMI. Higher BMI was associated with higher concentrations of C16:0, C18:0, C20:0, C22:0, and C24:1 in a middleaged population, ${ }^{38}$ and another study showed that C18:0, C20:0, and C24:1 were elevated in obese individuals who also had a diagnosis of Type 2 diabetes. ${ }^{39,40}$ Research shows that 
circulating free fatty acids are elevated in obese individuals, which serve as substrates for ceramide synthesis and could lead to an overproduction of ceramides. ${ }^{41}$ Once excess ceramides accumulate, C18:0 specifically has been shown to reduce fatty acid oxidation capacity, leading to accumulation and storage of fat. ${ }^{41}$ Thus, there appears to be a link bewteen obesity and increased production and accumulation of ceramides.

Ours was the first study to demonstrate that BMI is positively and significantly associated with the ceramide risk score. Even without participants in the two highest ceramide risk score categories, we found that participants in the moderate risk category had significantly higher ceramides than participants in the low risk category. This suggests that weight loss could decrease the ceramide risk score and thus reduce CVD risk, even in participants identified by the ceramide risk score as at moderate risk for CVD. Only one study has analyzed the influence of weight loss on ceramides, and this was through gastric bypass surgery in severely obese individuals. ${ }^{17}$ Multiple ceramide species were decreased in this population 3 months after surgery. ${ }^{17}$ While gastric bypass surgery is used only in severe cases of obesity, these results suggests that reducing BMI through less invasive measures, such as long-term dietary interventions, could potentially decrease ceramide accumulation and corresponding CVD risk.

\section{Diet}

While we did not see associations between ceramide species that make up the ceramide risk score or the risk score with diet quality after adjustments for confounding CVD risk factors, we did see that greater adherence to the HEI-2015 was associated with lower C22:0. There is limited research on the associations between diet quality measures and ceramides, and ours was the first study analyze the HEI-2015 and adherence to the U.S. DGAs, a dietary pattern known to reduce CVD risk. ${ }^{24,42}$ While C22:0 is not part of the ceramide risk score, previous literature shows it is positively associated with CVD risk ${ }^{22,43}$ and has been shown to be modifiable by diet. 
One dietary intervention on the Nordic Diet reported decreases in ceramide concentrations and specifically C22:0 after 12 weeks, but not after 24 weeks. ${ }^{21}$ Heilbronn et al. also observed elevated C22:0 in participants who were overfed 1,250 kilocalories per day. ${ }^{18}$ De novo ceramide synthesis depends on the availability of free fatty acids, specifically the saturated fat palmitate. ${ }^{6}$ Overconsumption of this saturated fat may result in excess ceramide accumulation , and lowering saturated fat consumption could perhaps be a target for dietary interventions to decrease ceramides. Future studies on the relationship between ceramides and diet quality are needed, specifically long-term intervention studies to assess changes in ceramides after improvements in diet quality.

When stratified by risk score category, we did not see an association between the HEI2015 and ceramide risk score categories. On average, participants in the moderate risk category had lower diet quality scores than participants in the low risk category, but this relationship was not statistically significant. We did not have participants in the high risk category, and only three participants in the increased risk category. We may have seen this trend continue and possibly become significant if we had a population with participants in the increased and high risk categories of the ceramide risk score.

\section{Covariates}

Similar to other studies, the most abundant serum ceramides in the body were C24:0, C24:1, and C22:0.44 Also similar to other studies, CVD biomarkers, which included LDL, HDL, and non-HDL cholesterol, were positively associated with multiple ceramides. ${ }^{13-15}$ Past smoking history was also associated with the ceramide risk score. Smoking is a risk factor for $\mathrm{CVD},{ }^{45}$ and even though we did not include current smokers, our results indicate that being a former smoker could be related to increased ceramides and CVD risk. Smoking has been shown to increase 
endothelial dysfunction, ${ }^{45}$ which is also a trademark of ceramides, leading to CVD progression. Additionally in our population, C24:0 was positively associated with LDL, non-HDL, and triglycerides, but inversely associated with fibrinogen, a clotting factor shown to be elevated in patients with CVD. ${ }^{46}$ Studies have suggested that C24:0 is not related to CVD risk, ${ }^{8}$ and further studies on the ceramide risk score have shown that C24:0 is not predictive of CVD. ${ }^{13,15,47}$ However, in two studies, C24:0 was elevated in patients with insulin resistance, ${ }^{39,40}$ a risk factor for CVD. Thus, C24:0's role in disease pathogenesis is still unclear and could possibly differ among disease states.

We did not see associations between statin use or hypertension and any of the ceramides or ceramide risk score. Ceramides involved in the ceramide risk score and the ceramide risk score itself have been studied in the context of their predictive capacity of CVD or cardiovascular end points and mortality in a number of studies. ${ }^{13,15,47,48}$ While our population did have at least one risk factor for CVD, we did not specifically seek out the participants with a diagnosis of CVD, whereas studies on this topic used populations who had diagnosed CVD. Overall, our population had lower serum ceramides than those reported in other studies that investigated these ceramides. ${ }^{13,15,47}$ Two large observational studies that used the ceramide risk score found that participants with a score of 10-12 (high risk) had a four-to six-fold increase in risk events compared to patients with a score of less than or equal to 2 points. Over half of our population had a risk score less than 2 points. This lack of variability among risk score categories may explain why we did not see ceramides or the ceramide risk score associated with statin use or hypertension, which has been seen in other studies. ${ }^{8,47}$

\section{Strengths and Limitations}


One strength of this study was the use of multiple $24-\mathrm{h}$ recalls performed by trained research personnel to estimate nutrient intake. We also analyzed and reported data on ceramides distinctly related to CVD and the ceramide risk score, which have not been studied alongside BMI and the HEI-2015. However, this study was not without limitations. Our sample size of 96 was small and a convenience sample. The majority of our participants were non-Hispanic white, which is characteristic of West Virginia but makes it difficult to generalize our findings to other populations. The cross-sectional study design does not allow us to look at long term changes in ceramides over time, inhibiting our ability to make conclusions about what factors can increase or decrease ceramide levels. Further, some of our data relied on self-report, such as dietary intake, medications, and medical conditions. There are inherent limitations with self-reported intake data, specifically under-reporting of diet intake ${ }^{49}$ or failing to report all medications used.

\section{Conclusion}

This study showed that middle-aged adults with obesity had higher circulating C18:0 and C18:0/24:0, and higher ceramide risk scores than those who were normal weight or overweight. Higher BMI remained independently associated with higher ceramide levels after adjusting for confounding variables. Future studies are needed to determine if reduction in weight status results in lower ceramide risk scores in humans, and if interventions to increase diet quality would be effective in lowering ceramides and thereby the ceramide risk score.

\section{Acknowledgements}

The authors would like to thank WVU research assistants' Paige Starrett, Brittany Abruzzino, Stephanie Thompson, and Michelle Campion for their contributions to data collection. They would also like to express their gratitude to the men and women who participated in the study. 
Author Contributions were as follows: M.V.M. designed the study, M.V.M conducted research; M.A.D. and I.H. analyzed the data; M.A.D. and M.V.M drafted the manuscript; all authors wrote and approved the final manuscript. 


\section{Tables and Figures}

Table 1. Participant characteristics stratified by ceramide risk category

\begin{tabular}{|c|c|c|c|c|}
\hline & $\begin{array}{c}\text { All } \\
\mathrm{N}=96\end{array}$ & $\begin{array}{c}\text { Low Risk } \\
n=56\end{array}$ & $\begin{array}{c}\text { Moderate Risk } \\
\qquad=\mathbf{4 0}\end{array}$ & p-value \\
\hline \multicolumn{5}{|l|}{ Demographic Factors } \\
\hline Age, y & $54.30 \pm 0.47$ & $53.95 \pm 0.55$ & $54.8 \pm 0.84$ & 0.40 \\
\hline Sex, women & $55(57.3 \%)$ & $33(58.9 \%)$ & $22(55.0 \%)$ & 0.70 \\
\hline \multicolumn{5}{|l|}{ Health-Related Factors } \\
\hline BMI $\left(\mathrm{kg} / \mathrm{m}^{2}\right)$ & $30.85 \pm 0.74$ & $29.22 \pm 0.92$ & $33.13 \pm 1.13$ & $0.003 *$ \\
\hline Elevated WC & $70(72.9 \%)$ & $37(66.1 \%)$ & $33(82.5 \%)$ & 0.07 \\
\hline Elevated WHR & $63(65.6 \%)$ & $36(64.3 \%)$ & $27(67.5 \%)$ & 0.74 \\
\hline FMI $\left(\mathrm{kg} / \mathrm{m}^{2}\right)$ & $12.26 \pm 0.55$ & $11.00 \pm 0.65$ & $14.02 \pm 0.89$ & $0.004 *$ \\
\hline Elevated Total Cholesterol & $52(54.2 \%)$ & $30(53.6 \%)$ & $22(55.0 \%)$ & 0.89 \\
\hline Elevated LDL & $77(81.1 \%)$ & $43(76.8 \%)$ & $34(87.2 \%)$ & 0.20 \\
\hline Low HDL & $22(22.9 \%)$ & $12(21.4 \%)$ & $10(25.0 \%)$ & 0.68 \\
\hline Elevated Triglycerides & $18(18.8 \%)$ & $9(16.1 \%)$ & $9(22.5 \%)$ & 0.43 \\
\hline Elevated Glucose & $35(36.5 \%)$ & $19(33.9 \%)$ & $16(40.0 \%)$ & 0.54 \\
\hline Elevated Insulin & $4(4.17 \%)$ & $1(1.8 \%)$ & $3(7.5 \%)$ & 0.17 \\
\hline HOMA-IR & $1.7(0.93-3.1)$ & $1.6(0.9-2.9)$ & $1.9(1.1-3.2)$ & 0.19 \\
\hline Elevated CRP & $16(16.7 \%)$ & $9(16.1 \%)$ & $7(17.5 \%)$ & 0.85 \\
\hline Elevated Fibrinogen & $22(22.9 \%)$ & $8(14.3 \%)$ & $14(35.0 \%)$ & $0.02 *$ \\
\hline Metabolic Syndrome & $37(38.5 \%)$ & $16(28.6 \%)$ & $21(52.5 \%)$ & $0.02 *$ \\
\hline Diabetes & $39(40.6 \%)$ & $22(39.3 \%)$ & $17(42.5 \%)$ & 0.75 \\
\hline Dyslipidemia & $88(91.7 \%)$ & $50(89.3 \%)$ & $38(95.0 \%)$ & 0.32 \\
\hline Hypertension & $37(38.5 \%)$ & $18(32.1 \%)$ & $19(47.5 \%)$ & 0.13 \\
\hline Statin use & $23(24.0 \%)$ & $13(23.2 \%)$ & $10(25.0 \%)$ & 0.84 \\
\hline \multicolumn{5}{|l|}{ Lifestyle Factors } \\
\hline Former Smoker & $48(50.0 \%)$ & $23(41.1 \%)$ & $25(62.5 \%)$ & $0.04 *$ \\
\hline HEI-2015 Diet Scores & $54.05 \pm 1.45$ & $55.27 \pm 1.73$ & $52.36 \pm 2.49$ & 0.34 \\
\hline
\end{tabular}

Values are means \pm SEM or frequency (percent). Abbreviations: BMI, body mass index; WC, waist circumference; WHR, waist-to-hip ratio; FMI, fat mass index; LDL, low density lipoprotein; HDL, high density lipoprotein; HOMA-IR, homeostatic model assessment of insulin resistance; CRP, c-reactive protein; HEI-2015, Healthy Eating Index 2015; LDL ( $\mathrm{n}=95$ ). Low risk, ceramide risk score 0-2; Moderate risk, ceramide risk score 3-6, including 3 participants with scores of 7 (2) and 8 (1), combined for descrip tive purposes. Student's t-test or Chi-Square tests were used to test significance. *Significant pvalue $<0.05$. 
Table 2. Bivariate analysis of ceramides by risk factors for cardiovascular disease to assess for possible covariates

\begin{tabular}{|c|c|c|c|c|c|c|c|c|c|c|}
\hline Risk Factor & C16:0 & C18:0 & C24:1 & C16:0/24:0 & C18:0/24:0 & C24:1/24:0 & C24:0 & Risk Score & C20:0 & C22:0 \\
\hline FMI & NS & $\begin{array}{c}0.37 ; 0.14 \\
(<0.001)\end{array}$ & NS & $\begin{array}{c}0.21 ; 0.08 \\
(0.003)\end{array}$ & $\begin{array}{c}0.53 ; 0.25 \\
(<0.001)\end{array}$ & $\begin{array}{c}0.17 ; 0.06 \\
(0.01)\end{array}$ & $\begin{array}{c}-0.15 ; 0.06 \\
(0.01)^{*}\end{array}$ & $\begin{array}{c}2.86 ; 0.05 \\
(0.01)\end{array}$ & NS & NS \\
\hline LDL & NS & NS & $\begin{array}{c}0.0009 ; 0.05 \\
(0.02)^{*}\end{array}$ & NS & NS & NS & $\begin{array}{c}0.001 ; 0.12 \\
(<0.001)^{*}\end{array}$ & NS & $\begin{array}{c}0.001 ; 0.04 \\
(0.03)^{*}\end{array}$ & $\begin{array}{c}0.001 ; 0.11 \\
(<0.001)^{*}\end{array}$ \\
\hline HDL & $\begin{array}{c}-0.35 ; 0.16 \\
(<0.001)^{*}\end{array}$ & $\begin{array}{c}-0.68 ; 0.21 \\
(<0.001)^{*}\end{array}$ & NS & $\begin{array}{c}-0.24 ; 0.04 \\
(0.02) \\
\end{array}$ & $\begin{array}{c}-0.58 ; 0.12 \\
(<0.001) \\
\end{array}$ & NS & NS & NS & $\begin{array}{c}-0.35 ; 0.06 \\
(0.007)\end{array}$ & $\begin{array}{c}-0.33 ; 0.07 \\
(0.004)\end{array}$ \\
\hline Non-HDL & $\begin{array}{c}0.25 ; 0.05 \\
(0.01)^{*}\end{array}$ & NS & $\begin{array}{l}0.47 ; 0.17 \\
(<0.001)^{*}\end{array}$ & $\begin{array}{c}-0.30 ; 0.05 \\
(0.02)\end{array}$ & NS & NS & $\begin{array}{l}0.55 ; 0.28 \\
(<0.001)^{*}\end{array}$ & NS & $\begin{array}{l}0.69 ; 0.20 \\
(<0.001)^{*}\end{array}$ & $\begin{array}{l}0.73 ; 0.30 \\
(<0.001)^{*}\end{array}$ \\
\hline Glucose & $\begin{array}{c}0.67 ; 0.07 \\
(0.008)^{*}\end{array}$ & $\begin{array}{c}1.34 ; 0.09 \\
(0.002)^{*}\end{array}$ & NS & $\begin{array}{c}0.92 ; 0.08 \\
(0.003)^{*}\end{array}$ & $\begin{array}{l}1.58 ; 0.11 \\
(<0.001)^{*}\end{array}$ & NS & NS & NS & NS & NS \\
\hline Fibrinogen & NS & NS & NS & $\begin{array}{l}0.45 ; 0.11 \\
(<0.001)^{*}\end{array}$ & $\begin{array}{c}0.63 ; 0.09 \\
(0.002)\end{array}$ & $\begin{array}{c}0.32 ; 0.06 \\
(0.01)\end{array}$ & $\begin{array}{c}-0.43 ; 0.14 \\
(<0.001)^{*}\end{array}$ & NS & NS & NS \\
\hline Smoking & NS & NS & NS & NS & NS & $\begin{array}{c}0.08 \\
(0.004)^{*}\end{array}$ & $\begin{array}{c}0.05 \\
(0.02)\end{array}$ & $\begin{array}{c}0.05 \\
(0.02)^{*}\end{array}$ & NS & $\begin{array}{c}0.05 \\
(0.02)\end{array}$ \\
\hline $\mathrm{WC}, \mathrm{cm}$ & NS & $\begin{array}{c}0.004 ; 0.18 \\
(<0.001)\end{array}$ & NS & $\begin{array}{c}0.002 ; 0.06 \\
(0.02)\end{array}$ & $\begin{array}{c}0.005 ; 0.22 \\
(<0.001)\end{array}$ & $\begin{array}{c}0.002 ; 0.05 \\
(0.03)\end{array}$ & NS & $\begin{array}{c}0.030 ; 0.06 \\
(0.02)\end{array}$ & NS & NS \\
\hline WHR, cm & NS & $\begin{array}{c}0.62 ; 0.08 \\
(0.006)\end{array}$ & NS & NS & NS & NS & NS & NS & NS & NS \\
\hline Triglycerides & NS & $\begin{array}{c}0.40 ; 0.15 \\
(<0.001)\end{array}$ & $\begin{array}{c}0.16 ; 0.07 \\
(0.01)\end{array}$ & NS & NS & NS & $\begin{array}{c}0.18 ; 0.10 \\
(0.003)\end{array}$ & NS & $\begin{array}{c}0.40 ; 0.22 \\
(<0.001)\end{array}$ & NS \\
\hline Insulin & NS & $\begin{array}{c}0.23 ; 0.19 \\
(<0.001)\end{array}$ & NS & $\begin{array}{c}0.08 ; 0.04 \\
(0.04)\end{array}$ & $\begin{array}{c}0.26 ; 0.20 \\
(<0.001)\end{array}$ & NS & NS & $\begin{array}{c}1.44 ; 0.05 \\
(0.02)\end{array}$ & $\begin{array}{c}0.15 ; 0.10 \\
(0.002)\end{array}$ & NS \\
\hline HOMA-IR & $\begin{array}{c}0.07 ; 0.06 \\
(0.02)\end{array}$ & $\begin{array}{c}0.23 ; 0.18 \\
(<0.001) \\
\end{array}$ & NS & $\begin{array}{c}0.10 ; 0.06 \\
(0.02)\end{array}$ & $\begin{array}{c}0.25 ; 0.18 \\
(<0.001)\end{array}$ & NS & NS & NS & $\begin{array}{c}0.15 ; 0.10 \\
(0.002)^{*}\end{array}$ & NS \\
\hline CRP & NS & $\begin{array}{c}0.12 ; 0.07 \\
(0.01)\end{array}$ & NS & NS & $\begin{array}{c}0.15 ; 0.09 \\
(0.004)\end{array}$ & NS & NS & NS & NS & NS \\
\hline Diabetes & NS & $\begin{array}{c}0.06 \\
(0.006) \\
\end{array}$ & NS & NS & $\begin{array}{c}0.04 \\
(0.03) \\
\end{array}$ & NS & NS & NS & NS & NS \\
\hline MetS & NS & $\begin{array}{c}0.06 \\
(0.008)\end{array}$ & NS & NS & $\begin{array}{c}0.09 \\
(<0.001)\end{array}$ & NS & NS & NS & NS & NS \\
\hline
\end{tabular}

Values reported are slope per unit change; coefficient of determination $\left(\mathrm{R}^{2}\right)\left(\mathrm{p}\right.$-value). For smoking, diabetes, and MetS, $\mathrm{R}^{2}$ and $\mathrm{p}$-value reported. *p-values significant in bivariate analysis that remained significant after stepwise regression and were later included in the adjusted model. Abbreviations: FMI, fat mass index; CHL, cholesterol; LDL, low density lipoprotein; HDL, high density lipoprotein; WC, waist circumference; WHR, waist-to-hip ratio; HOMA-IR, homeostatic model assessment of insulin resistance; CRP, c-reactive protein; MetS, Metabolic Syndrome; NS, non-significant. Variables not significant with any ceramides include: age, gender, income, statin use, and hypertension. 
Table 3. Associations in HEI-2015 and BMI with ceramides and ceramide risk score

\begin{tabular}{|c|c|c|c|c|c|c|c|}
\hline \multirow{2}{*}{$\begin{array}{l}\text { Outcome } \\
\text { Variables }\end{array}$} & \multicolumn{4}{|c|}{ Ceramides Included in the Ceramide Risk Score } & \multirow{2}{*}{$\begin{array}{l}\text { Ceramide } \\
\text { Risk Score }\end{array}$} & \multicolumn{2}{|c|}{ Other Ceramides } \\
\hline & $\mathrm{C} 18: 0$ & $\mathrm{C} 24: 1$ & $\mathrm{C} 16: 0 / 24: 0$ & $\mathrm{C} 18: 0 / 24: 0$ & & $\mathrm{C} 20: 0$ & $\mathrm{C} 22: 0$ \\
\hline \multicolumn{8}{|c|}{ Unadjusted Model } \\
\hline HEI-2015 & NS & NS & NS & NS & NS & NS & $\begin{array}{c}-0.002 ; 0.06 \\
(0.009)\end{array}$ \\
\hline $\operatorname{BMI}\left(\mathrm{kg} / \mathrm{m}^{2}\right)$ & $\begin{array}{c}0.80 ; 0.19 \\
(<0.001)\end{array}$ & NS & $\begin{array}{c}0.36 ; 0.07 \\
(0.006)\end{array}$ & $\begin{array}{c}1.02 ; 0.26 \\
(<0.001)\end{array}$ & $\begin{array}{c}5.97 ; 0.07 \\
(0.006)\end{array}$ & NS & NS \\
\hline \multicolumn{8}{|l|}{ Adjusted Models } \\
\hline HEI-2015 & NS & NS & NS & NS & NS & NS & $\begin{array}{c}-0.002 ; 0.42 \\
(0.02)\end{array}$ \\
\hline $\operatorname{BMI}\left(\mathrm{kg} / \mathrm{m}^{2}\right)$ & $\begin{array}{l}0.81 ; 0.31 \\
(<0.0001)\end{array}$ & NS & NS & $\begin{array}{l}0.91 ; 0.30 \\
(<0.0001)\end{array}$ & $\begin{array}{c}5.58 ; 0.11 \\
(0.009)\end{array}$ & NS & NS \\
\hline
\end{tabular}

Values reported are slope per unite change; coefficient of determination $\mathrm{R}^{2}$ and (p-value). Abbreviations: HEI-2015, Healthy Eating Index 2015; BMI, Body Mass Index; NS, not significant. Covariates used in adjusted models varied by ceramide: C18:0 (HDL-C, glucose); C24:1 (LDL, non-HDL); C16:0/24:0 (total cholesterol, glucose, fibrinogen); C1 8:0/24:0 (glucose); C24:1/24:0 (smoking); ceramide risk score (smoking); C20:0 (LDL, non-HDL); C22:0 (LDL, non-HDL). 


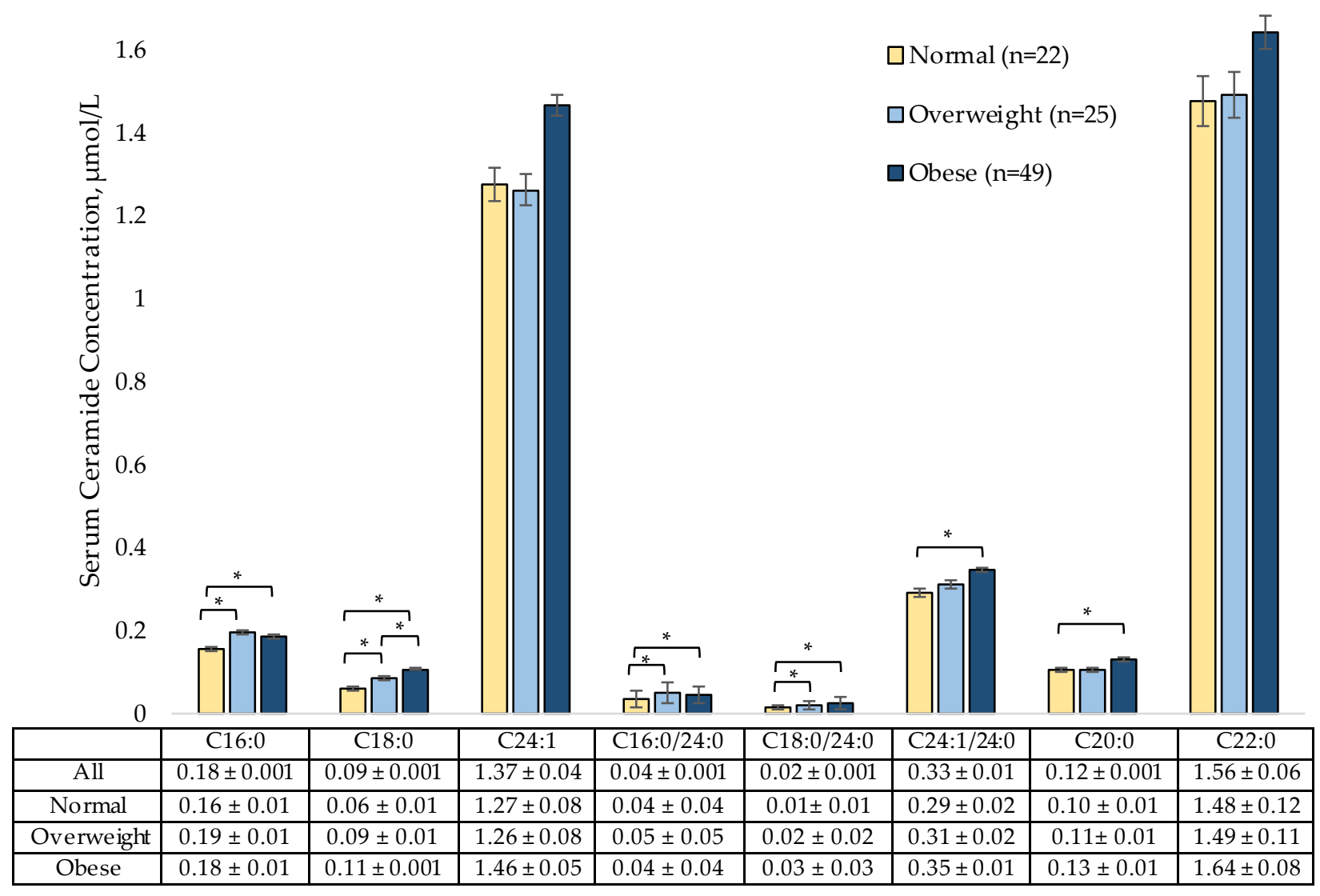

Figure 1. Serum ceramide concentrations in $\mu \mathrm{mol} / \mathrm{L}$ by BMI categories normal $\left(18.5-25 \mathrm{~kg} / \mathrm{m}^{2}\right)$, overweight $(25-30$ $\mathrm{kg} / \mathrm{m}^{2}$ ), and obese (over $30 \mathrm{~kg} / \mathrm{m}^{2}$ ). Me ans and SEM of ce ra mide concentrations reported. Tukey's HSD use do test significance between the three categories. *Significant p-values less than 0.05 


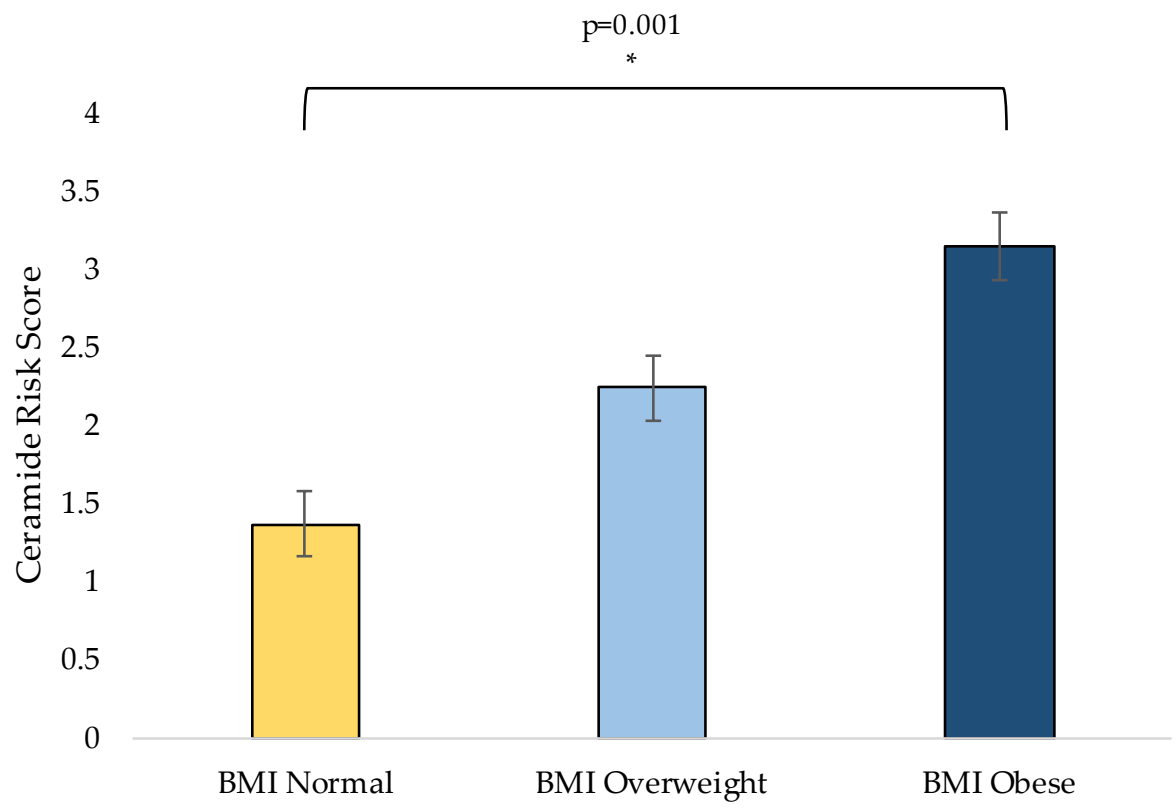

Figure 2. Ceramide riskscore by BMI categories normal $\left(18.5-25 \mathrm{~kg} / \mathrm{m}^{2}\right)$, overweight $\left(25-30 \mathrm{~kg} / \mathrm{m}^{2}\right)$, and obe se (over $30 \mathrm{~kg} / \mathrm{m}^{2}$ ). Me ans and SEM for ceramide risk score by BMI categories: normal $(1.36 \pm 0.41)$, overweight $(2.24 \pm$ $0.38)$, and obese $(3.14 \pm 0.27)$. Tukey's HSD used to test significance between the three categories. ${ }^{*}$ Significant $\mathrm{p}$-value $=0.001$ 
Supplemental Table.HEI-2015 criteria for scoring

\begin{tabular}{|c|c|c|c|}
\hline Component & Max Score & Stan dard for max score & Standard for minimum score $(0)$ \\
\hline TotalFruits & 5 & $\geq 0.8$ cup eq $1,000 \mathrm{kcal}$ & No fruit \\
\hline Whole Fruits & 5 & $\geq 0.4$ cup eq $/ 1,000 \mathrm{kcal}$ & No whole fruit \\
\hline Total Veget ables & 5 & $\geq 1.1$ cup eq $/ 1,000 \mathrm{kcal}$ & No vegetables \\
\hline Greens and Beans & 5 & $\geq 0.2$ cup eq $1,000 \mathrm{kcal}$ & No dark-green vegetables or legumes \\
\hline Whole Grains & 10 & $\geq 1.5 \mathrm{oz} \mathrm{eq} / 1,000 \mathrm{kcal}$ & No whole grains \\
\hline Dairy & 10 & $\geq 1.3 \mathrm{cup} \mathrm{eq} / 1,000 \mathrm{kcal}$ & No dairy \\
\hline Total Protein Foods & 5 & $\geq 2.5 \mathrm{oz}$ eq $/ 1,000 \mathrm{kcal}$ & No protein foods \\
\hline Seafood and Plant Proteins & 5 & $\geq 0.8 \mathrm{oz} \mathrm{eq} / 1,000 \mathrm{kcal}$ & No seafood or plant proteins \\
\hline Fatty Acids & 10 & (PUFAS+MUFAS)/SFAs $\geq 2.5$ & (PUFAs+MUFAs)/SFAs $\leq 1.2$ \\
\hline Refined Grains & 10 & $\leq 1.8 \mathrm{oz} \mathrm{eq} / 1,000 \mathrm{kcal}$ & $\geq 4.3 \mathrm{oz} \mathrm{eq} / 1,000 \mathrm{kcal}$ \\
\hline Sodium & 10 & $\leq 1.1 \mathrm{gram} / 1,000 \mathrm{kcal}$ & $\geq 2.0 \mathrm{oz} \mathrm{eq} / 1,000 \mathrm{kcal}$ \\
\hline Added Sugars & 10 & $\leq 6.5 \%$ of energy & $\geq 26 \%$ of energy \\
\hline Saturated Fats & 10 & $\leq 8 \%$ of energy & $\geq 16 \%$ of energy \\
\hline
\end{tabular}

Abbreviations: HEI-2015, Healthy Eating Index 2015; PUFA, polyunsaturated fatty acids; MUFA, monounsaturated fatty acids; SFA, saturated fatty acids 


\section{CHAPTER 3: CONCLUSION}

As ceramides are being clinically tested as a marker of CVD risk, it is important to understand what factors contribute to elevated ceramide levels in order to formulate effective intervention strategies to decrease ceramides. Our objective was to study the associations between ceramides, diet quality, and modifiable risk factors for CVD such as obesity, specifically through BMI. The current study shows that BMI is more predictive of ceramide concentration than diet quality in this sample of middle-aged adults at risk for CVD. Future studies are needed to determine if reduction in weight status results in lower ceramide risk scores in humans, and if interventions to increase diet quality would be effective in lowering ceramides and thereby the ceramide risk score. 


\section{REFERENCES}

1. WHO | Cardiovascular diseases (CVDs). WHO. http:/www.who.int/cardiovascular_diseases/en/. Accessed November 1, 2018.

2. Centers for Disease Control and Prevention. Heart Disease Facts \& Statistics $\mid$ cdc.gov. https:/www.cdc.gov/heartdisease/facts.htm. Published October 9, 2018. Accessed October 24, 2018.

3. Vasan Ramachandran S. Biomarkers of Cardiovascular Disease. Circulation. 2006;113(19):2335-2362. doi:10.1161/CIRCULATIONAHA.104.482570

4. Sachdeva A, Cannon CP, Deedwania PC, et al. Lipid levels in patients hospitalized with coronary artery disease: an analysis of 136,905 hospitalizations in Get With The Guidelines. Am Heart J. 2009;157(1):111-117.e2. doi:10.1016/j.ahj.2008.08.010

5. Mundra PA, Shaw JE, Meikle PJ. Lipidomic analyses in epidemiology. Int J Epidemiol. 2016;45(5):1329-1338. doi:10.1093/ije/dyw112

6. Bikman BT, Summers SA. Ceramides as modulators of cellular and whole-body metabolism. J Clin Invest. 2011;121(11):4222-4230. doi:10.1172/JCI57144

7. Bismuth J, Lin P, Yao Q, Chen C. Ceramide: a common pathway for atheroscleros is? Atherosclerosis. 2008;196(2):497-504. doi:10.1016/j.atherosclerosis. 2007.09.018

8. Tarasov K, Ekroos K, Suoniemi M, et al. Molecular lipids identify cardiovascular risk and are efficiently lowered by simvastatin and PCSK9 deficiency. J Clin Endocrinol Metab. 2014;99(1):E45-52. doi:10.1210/jc.2013-2559

9. Menuz V, Howell KS, Gentina S, et al. Protection of C. elegans from anoxia by HYL-2 ceramide synthase. Science. 2009;324(5925):381-384. doi:10.1126/science.1168532

10. Park Y-MM, Fung TT, Steck SE, et al. Diet Quality and Mortality Risk in Metabolically Obese Normal-Weight Adults. Mayo Clin Proc. 2016;91(10):1372-1383.

doi:10.1016/j.mayocp.2016.06.022

11. Turpin SM, Nicholls HT, Willmes DM, et al. Obesity-Induced CerS6-Dependent C16:0 Ceramide Production Promotes Weight Gain and Glucose Intolerance. Cell Metabolism. 2014;20(4):678-686. doi:10.1016/j.cmet.2014.08.002

12. Mayo Clinic. Plasma Ceramides: A Novel Biomarker of Unstable Atherosclerotic Cardiovascular Disease.; :4. https://cdn.prod-

carehubs.net/n1/96e99366cea7b0de/uploads/2016/07/ceramides-brochure-final-0616.pdf.

13. Meeusen JW, Donato LJ, Bryant SC, Baudhuin LM, Berger PB, Jaffe AS. Plasma Ceramides: A Novel Predictor of Major Adverse Cardiovascular Events After Coronary Angiography. Arterioscler Thromb Vasc Biol. June 2018. doi:10.1161/ATVBAHA.118.311199 
14. Laaksonen R, Ekroos K, Sysi-Aho M, et al. Plasma ceramides predict cardiovascular death in patients with stable coronary artery disease and acute coronary syndromes beyond LDLcholesterol. Eur Heart J. 2016;37(25):1967-1976. doi:10.1093/eurheartj/ehw148

15. Havulinna AS, Sysi-Aho M, Hilvo M, et al. Circulating Ceramides Predict Cardiovascular Outcomes in the Population-Based FINRISK 2002 Cohort. Arterioscler Thromb Vasc Biol. 2016;36(12):2424-2430. doi:10.1161/ATVBAHA.116.307497

16. Bergman BC, Brozinick JT, Strauss A, et al. Serum sphingolipids: relationships to insulin sensitivity and changes with exercise in humans. Am J Physiol Endocrinol Metab. 2015;309(4):E398-408. doi:10.1152/ajpendo.00134.2015

17. Huang H, Kasumov T, Gatmaitan P, et al. Gastric bypass surgery reduces plasma ceramide subspecies and improves insulin sensitivity in severely obese patients. Obesity (Silver Spring). 2011;19(11):2235-2240. doi:10.1038/oby.2011.107

18. Heilbronn LK, Coster ACF, Campbell LV, et al. The effect of short-term overfeeding on serum lipids in healthy humans. Obesity (Silver Spring). 2013;21(12):E649-659. doi:10.1002/oby.20508

19. Luukkonen PK, Sädevirta S, Zhou Y, et al. Saturated Fat Is More Metabolically Harmful for the Human Liver Than Unsaturated Fat or Simple Sugars. Diabetes Care. 2018;41(8):1732-1739. doi:10.2337/dc18-0071

20. Kien CL, Bunn JY, Poynter ME, et al. A lipidomics analysis of the relationship between dietary fatty acid composition and insulin sensitivity in young adults. Diabetes. 2013;62(4):1054-1063. doi:10.2337/db12-0363

21. Lankinen M, Schwab U, Kolehmainen M, et al. A Healthy Nordic Diet Alters the Plasma Lipidomic Profile in Adults with Features of Metabolic Syndrome in a Multicenter Randomized Dietary Intervention. J Nutr. 2016;146(4):662-672. doi:10.3945/jn.115.220459

22. Wang DD, Toledo E, Hruby A, et al. Plasma Ceramides, Mediterranean Diet, and Incident Cardiovascular Disease in the PREDIMED Trial. Circulation. March 2017:CIRCULATIONAHA.116.024261. doi:10.1161/CIRCULATIONAHA.116.024261

23. Loprinzi PD, Branscum A, Hanks J, Smit E. Healthy Lifestyle Characteristics and Their Joint Association With Cardiovascular Disease Biomarkers in US Adults. Mayo Clin Proc. 2016;91(4):432-442. doi:10.1016/j.mayocp.2016.01.009

24. Reedy J, Krebs-Smith SM, Miller PE, et al. Higher diet quality is associated with decreased risk of all-cause, cardiovascular disease, and cancer mortality among older adults. J Nutr. 2014;144(6):881-889. doi:10.3945/jn.113.189407

25. Harris PA, Taylor R, Thielke R, Payne J, Gonzalez N, Conde JG. Research electronic data capture (REDCap) - A metadata-driven methodology and workflow process for providing translational research informatics support. Journal of Biomedical Informatics. 2009;42(2):377-381. doi:10.1016/j.jbi.2008.08.010 
26. National Institute of Health. Diabetes \& Prediabetes Tests | NIDDK. National Institute of Diabetes and Digestive and Kidney Diseases. https:/www.niddk.nih.gov/healthinformation/diagnostic-tests/diabetes-prediabetes. Accessed July 30, 2018.

27. National Institute of Health. High Blood Pressure. National Institute on Aging. http://www.nia.nih.gov/health/high-blood-pressure. Accessed July 30, 2018.

28. Grundy SM, H. Bryan Brewer J, Cleeman JI, Sidney C. Smith J, Lenfant C. Definition of Metabolic Syndrome. Circulation. 2004;109:433-438. doi:10.1161/01.CIR.0000111245.75752.C6

29. Ostchega Y, Nwankwo T, Sorlie PD, Wolz M, Zipf G. Assessing the validity of the Omron HEM-907XL oscillometric blood pressure measurement device in a National Survey environment. J Clin Hypertens (Greenwich). 2010;12(1):22-28. doi:10.1111/j.17517176.2009.00199.x

30. Viera AJ, Zhu S, Hinderliter AL, Shimbo D, Person SD, Jacobs DR. Diurnal blood pressure pattern and development of prehypertension or hypertension in young adults: the CARDIA study. J Am Soc Hypertens. 2011;5(1):48-55. doi:10.1016/j.jash.2010.12.002

31. National Heart, Lung, and Blood Institute. Classification of Overweight and Obesity by BMI, Waist Circumference, and Associated Disease Risks. https $/ /$ www.nhlbi.nih. gov/health/educational/lose_wt/BMI/bmi_dis.htm. Published August 16, 2017. Accessed August 16, 2017.

32. National Cancer Institute Division of Cancer Control and Population Sciences. The Healthy Eating Index Research Uses: Overview of the Methods \& Calculations.

https:/epi.grants.cancer.gov/hei/hei-methods-and-calculations.html. Accessed July 2, 2018.

33. U.S. Department of Health and Human Services and U.S. Department of Agriculture. 2015-2020 Dietary Guidelines for Americans - health.gov. https:/health.gov/dietaryguidelines/2015/. Accessed June 28, 2018.

34. Nutrition Coordination Center (NCC), University of Minnesota. Guide to Creating Variables, Needed to Calculate Scores for Each Component of the Healthy Eating Index2015 (HEI-2015). http $/ /$ www.ncc.umn.edu/ndsrsupport/hei2015.pdf. Accessed August 16, 2017.

35. Rico JE, Bandaru VVR, Dorskind JM, Haughey NJ, McFadden JW. Plasma ceramides are elevated in overweight Holstein dairy cows experiencing greater lipolysis and insulin resistance during the transition from late pregnancy to early lactation. Journal of Dairy Science. 2015;98(11):7757-7770. doi:10.3168/jds.2015-9519

36. Haughey NJ, Cutler RG, Tamara A, et al. Perturbation of sphingolipid metabolism and ceramide production in HIV-dementia. Ann Neurol. 2004;55(2):257-267. doi:10.1002/ana.10828 
37. Meikle PJ, Wong G, Barlow CK, et al. Plasma lipid profiling shows similar associations with prediabetes and type 2 diabetes. PLoS ONE. 2013;8(9):e74341.

doi:10.1371/journal.pone.0074341

38. Mielke MM, Bandaru VVR, Han D, et al. Demographic and clinical variables affecting mid- to late-life trajectories of plasma ceramide and dihydroceramide species. Aging Cell. 2015;14(6):1014-1023. doi:10.1111/acel.12369

39. Boon J, Hoy AJ, Stark R, et al. Ceramides contained in LDL are elevated in type 2 diabetes and promote inflammation and skeletal muscle insulin resistance. Diabetes. 2013;62(2):401-410. doi:10.2337/db12-0686

40. Haus JM, Kashyap SR, Kasumov T, et al. Plasma ceramides are elevated in obese subjects with type 2 diabetes and correlate with the severity of insulin resistance. Diabetes. 2009;58(2):337-343. doi:10.2337/db08-1228

41. Fucho R, Casals N, Serra D, Herrero L. Ceramides and mitochondrial fatty acid oxidation in obesity. FASEB J. 2017;31(4):1263-1272. doi:10.1096/fj.201601156R

42. Recommended Dietary Pattern to Achieve Adherence to the American Heart Association/American College of Cardiology (AHA/ACC) Guidelines: A Scientific Statement From the American Heart Association | Circulation. https:/www.ahajournals.org/doi/full/10.1161/CIR.0000000000000462. Accessed October $16,2018$.

43. Mantovani A, Bonapace S, Lunardi G, et al. Association between plasma ceramides and inducible myocardial ischemia in patients with established or suspected coronary artery disease undergoing myocardial perfusion scintigraphy. Metab Clin Exp. 2018;85:305-312. doi:10.1016/j.metabol.2018.05.006

44. Ichi I, Nakahara K, Miyashita Y, et al. Association of ceramides in human plasma with risk factors of atherosclerosis. Lipids. 2006;41(9):859-863. doi:10.1007/s11745-006-5041-6

45. Messner B, Bernhard D. Smoking and cardiovascular disease: mechanisms of endothelial dysfunction and early atherogenesis. Arterioscler Thromb Vasc Biol. 2014;34(3):509-515. doi:10.1161/ATVBAHA.113.300156

46. Stec JJ, Silbershatz H, Tofler GH, et al. Association of fibrinogen with cardiovascular risk factors and cardiovascular disease in the Framingham Offspring Population. Circulation. 2000;102(14):1634-1638.

47. Laaksonen R, Ekroos K, Sysi-Aho M, et al. Plasma ceramides predict cardiovascular death in patients with stable coronary artery disease and acute coronary syndromes beyond LDLcholesterol. Eur Heart J. 2016;37(25):1967-1976. doi:10.1093/eurheartj/ehw148

48. Anroedh S, Hilvo M, Akkerhuis KM, et al. Plasma concentrations of molecular lipid species predict long-term clinical outcome in coronary artery disease patients. J Lipid Res. 2018;59(9):1729-1737. doi:10.1194/jlr.P081281 
49. Subar AF, Freedman LS, Tooze JA, et al. Addressing Current Criticism Regarding the Value of Self-Report Dietary Data. J Nutr. 2015;145(12):2639-2645.

doi:10.3945/jn.115.219634 